\title{
Revisiting gradient wind balance in tropical cyclones using dropsonde observations
}

\author{
Jorge L. García-Franco ${ }^{\mathrm{a}, \mathrm{b} *}$ and Juliane Schwendike ${ }^{\mathrm{a}}$ \\ a School of Earth and Environment, University of Leeds, UK \\ ${ }^{\mathrm{b}}$ Atmospheric, Oceanic and Planetary Physics, Department of Physics. University of Oxford. \\ ${ }^{*}$ Correspondence to: Clarendon Laboratory, University of Oxford, Parks Road, Oxford OX1 3PU, United Kingdom. E-mail: \\ jorge.garcia-franco@physics.ox.ac.uk
}

This study diagnoses the degree of gradient wind balance (GWB) in dropsonde observations of 30 tropical cyclones (TCs) divided into 91 intense observation periods. The diagnosed GWB in these observation periods are composited to investigate which characteristics of a TC are significantly related to departures from GWB. This analysis confirms that on average the flow above the boundary layer is approximately in GWB. Supergradient flow is more common near the radius of maximum wind (RMW) in the upper boundary layer than above in the free troposphere or outside the RMW and is also more common in strong storms than in weak storms. In contrast, the degree of GWB does not differ between intensifying, steady-state and weakening storms. Storms with a peaked wind profile have a higher probability of showing supergradient winds than those with a flat wind profile. The comparison of two commonly used functions to fit observations shows that the diagnosing GWB from dropsonde observations is highly dependent on the analysis technique. The agradient wind magnitude and even sign is shown to depend on which of these functions is used to fit the observations. The use of a polynomial fit consistently diagnoses the presence of supergradient winds far more frequently than a piece-wise function, and also shows a marked degree of imbalance above the boundary layer. Therefore, caution is warranted when determining the degree of GWB with a polynomial fit.

This article has been accepted for publication and undergone full peer review but has not been through the copyediting, typesetting, pagination and proofreading process which may lead to differences between this version and the Version of Record. Please cite this article as doi: $10.1002 /$ qj.3947 
Gradient wind balance (GWB) in tropical cyclones (TCs) has long been investigated by observational and modelling studies (e.g. Gray

4 and unbalanced dynamics have for storm structure and intensification in theoretical and numerical models (Shapiro and Willoughby

5 1982; Bui et al. 2009; Smith and Montgomery 2010; Montgomery and Smith 2017; Heng et al. 2017). The first analyses of aircraft

6 data that diagnosed whether the wind was close to GWB found contradictory results because different methodologies would render

7 different conclusions (e.g. Gray 1962; Gray and Shea 1973; Willoughby 1979; Jorgensen 1984).

8 The debate remained until the influential analysis of Willoughby (1990) concluded that the mid- and low-troposphere of the majority TCs was in GWB. Although this result was contested by Gray (1991), GWB was considered a fair assumption in TCs and became common constraint in theoretical TC models, particularly above the boundary layer, for example in the Wind-Induced Surface 1. Heat Exchange (WISHE) model (e.g. Emanuel 1997) or in the Sawyer-Eliassen equation (Pendergrass and Willoughby 2009; Heng 12 et al. 2017). However, recent observations have shown that imbalances may occur. In particular, in dropwindsondes, several analyses 13 documented supergradient winds (e.g. Kepert 2006a,b; Schwendike and Kepert 2008; Bell and Montgomery 2008; Montgomery et al. 14 2014, hereafter M14), especially in the upper boundary layer.

The boundary layer model of Kepert (2001) and Kepert and Wang (2001) provides a theoretical framework to explain supergradient winds in the upper boundary layer. In their model, supergradient flow in the upper boundary layer was caused by the strong inward 17 advection of angular momentum, thereby linking the presence of supergradient winds to the radial distribution of angular momentum. 18 Furthermore, Stern et al. (2020) recently used an axisymmetric version of the boundary layer model of Kepert and Wang (2001) to 19 Xplain why the flow right above the top of the boundary layer should be weakly subgradient. Stern et al. (2020) argue that the layer of co ubgradient flow near the eyewall is associated with an inertial oscillation and is strongly influenced by the radius of maximum wind 21 (RMW).

Kepert (2006a,b) found that the degree of GWB in Hurricanes Georges (1998) and Mitch (1998) was a function of the radial shape of vind profile, which is related to the radial distribution of absolute angular momentum. Although Georges and Mitch had relatively 24 imilar intensities, the GWB analysis showed that Georges was close to GWB whereas Mitch showed markedly supergradient flow 25 near the RMW. One of the main differences between Mitch and Georges was the radial gradient of wind speed: while Georges had a mooth flat radial wind profile, Mitch had a peaked profile. These studies argue that the radial shape of the wind profile determines the 27 dvection of angular momentum and influence the degree of GWB in these storms, as accounted for by the model of Kepert and Wang 28 (2001).

Schwendike and Kepert (2008) provide further evidence that the shape of the radial wind profile is related to the degree of GWB. so They showed that Hurricane Danielle (1998) with relatively weak winds (Cat. 2) and a flat radial wind profile was close to GWB. In 31 contrast, their analysis of Hurricane Isabel (2003), a major hurricane (Cat. 5) with a peaked wind profile, showed strongly supergradient 32 winds in the upper boundary layer and was balanced above $2000 \mathrm{~m}$.

33 A recent theoretical examination of the validity of GWB arose in the paradigm for TC intensification, known as the boundary layer 34 spin-up mechanism, which proposes that supergradient flow in the boundary layer imports air into the eyewall with minimal loss of momentum (Smith and Montgomery 2010; Montgomery and Smith 2017). The supergradient flow in the boundary layer is advected upwards and acts to direcly intensify the vortex (e.g. Smith and Montgomery 2016; Montgomery and Smith 2017). Rogers et al. 2015; Abarca et al. 2016) has supported this theory by documenting supergradient winds in the boundary layer in 
50 equation. The comparison of these wind (and/or pressure) values estimated through the GWB equation with the observed tangential 51 ind (pressure), provided a measure of the degree of GWB.

The principal difference between the studies mentioned above was the analytical function used to fit the observations. For example, 1 Kepert (2006a) used a piece-wise function (Willoughby et al. 2006) whereas Bell and Montgomery (2008) and Didlake and Houze 2011) used a polynomial function (see Section 2). While M14 acknowledged that using polynomial fits could be too simplistic, they argued that a polynomial function was appropriate since the selection of this family of functions over other functions that they tested did not significantly affect the GWB analysis. However, several studies have discussed and shown that the analysis technique may be 57 key and potentially a source of error in determining balance in observations (Willoughby 1990; Gray 1991; Kepert 2006a).

58 In short, observational studies have recurrently found supergradient winds in TCs, and several theories and models of intensification 9 provide physical accounts for the wind flow to depart from GWB. The observational evidence of imbalances summarised above was (1) provided in single case studies. However, the question remains as to how many storms show a departure from GWB or indeed 61 which characteristics of a storm are linked to the degree of GWB. For instance, the boundary layer spin-up mechanism argues that 62 supergradient winds are an essential component of TC intensification, a feature that is supported by several dropsonde case studies 3 Yell and Montgomery 2008; Bell et al. 2012; Sanger et al. 2014; Rogers et al. 2015). One might reasonably infer from their evidence that there would be a relationship between the degree to which the winds are supergradient and the intensification rate, with intensifying 65 orms being more likely supergradient in the boundary layer and weakening storms being in GWB or having slight supergradient flow. S6 Similarly, the boundary layer model of Kepert and Wang (2001) suggested that the radial distribution of momentum determines the 67 location and magnitude of supergradient winds, which is also supported by dropsonde evidence in a handful of cases (Kepert 2006a,b; Schwendike and Kepert 2008). A reasonable hypothesis arising from their results is that storms with peaked wind profile will more $69 \sqrt{1}$ kely show supergradient signatures than storms with a flat wind profile.

This study investigates the degree of gradient wind balance in a larger set of TCc and dropsonde observations. The primary motivation of this study is to expand on the results from the case studies to quantify whether there is any statistical relation between TC characteristics and the degree of GWB. In particular, as explained above, four characteristics of TC structure and development have been theoretically linked to GWB: mean intensity, the rate of change of intensity, the height level and the shape of the radial wind profile.

This paper is structured as follows. Section 2 describes the data and the methodologies used to analyse the GWB and to composite the observations. Section 3 shows a few well-known example cases to illustrate and compare existing methods to measure the degree of GWB. Section 4 then presents the analysis of the degree of GWB in dropsonde composites. These results are discussed in Section 
88 sonde, accelerated descent rates and other system failures. The raw dropsonde files have been processed by the Atmospheric Sounding 89 Processing Environment (ASPEN) which smooths and filters the data, after which most dropsonde profiles were visually evaluated as urther quality control (Wang et al. 2015). In order to standardize the analysis, the GWB analysis was done in 100-m intervals from 100 - $\sqrt{91} \mathrm{~m}$ to the highest recorded level from each profile. The wind or pressure values in this 100-m spacing are obtained by averaging all the 1 92 data-points in a 20-m window. Previous studies such as Kepert (2006a,b) and Schwendike and Kepert (2008) accounted for the radial $\gamma_{93}$ notion of the sonde and the horizontal temperature gradient. The data in this study has not undergone these corrections. Other than the $\longrightarrow_{95}$ as vertical averaging to a 100-m interval grid as described above, the dropsonde profiles are only processed by putting the observations in storm relative-framework using the tracks described in the following section.

\section{Track determination}

(7) In accurate track can be essential for studies investigating the tangential wind speed in the inner-core (Kepert 2005), particularly in 98 regard to GWB assessments, due to the sensitivity of the analysis technique to track errors. Flight-level data (Vigh et al. 2015), available 99 99 at https://verif.rap.ucar.edu/tcdata/flight/, provide a track time-series with high temporal resolution. The tracks So in this dataset were estimated using the Willougby-Chelmow (WC) (Willoughby et al. 1982) algorithm, based on flight-level wind data. (141) The WC method minimises the distance between the centre of the storm and tangential lines to the direction of each wind observation. 102 This centre-finding method has been extensively used in dropsonde analyses (e.g. Zhang et al. 2011, 2013; Rogers et al. 2013) due to its (n) imple interpretation and application, as well as its suitability to be used on high temporal resolution onboard radar wind measurements $104)$ Zhang et al. 2011).

105 Although the main track finding algorithm used is the WC, two alternative track datasets were used to analyse the sensitivity of 106 the GWB analysis technique to the track: the best track dataset (Landsea and Franklin 2013) and the translating pressure fit (TPF) 107 technique (Kepert 2005). The best-track dataset (Landsea and Franklin 2013) has a 6-h temporal resolution which makes this dataset less suitable for inner-core studies but it has been extensively used, for instance, to determine the rate of intensity change of a storm (e.g. Hendricks et al. 2010). The TPF method determines the centre as the point that minimizes the error of fitting a profile (Holland 1980) to pressure observations (Kepert 2005).

\subsection{Intense observation periods}

An intense observation period (IOP) is defined as a finite time-frame where a storm was well-sampled. IOPs have been used previously (e.g. Rogers et al. 2013) to merge observations into a single sample and to evaluate the mean characteristics of a storm during a specific period. In particular, this study considers an IOP as a period where a given storm had more than ten dropsondes in the inner-core region (r $r<100 \mathrm{~km}$ ). Similarly, each IOP could be no longer than 12 hours and could not overlap with another IOP. This threshold 
allenging as in most of these cases the radial pressure gradient was very small which caused the GWB analysis to diagnose unrealistic radient wind estimations. Table 1 shows the information of each IOP, including storm name, start and end dates of each IOP.

\section{Compositing}

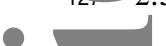

he IOPs were composited according to three main characteristics: mean TC intensity, intensity change rate, and the shape of the wind Son rofile. The analysed TCs were classified using the Saffir-Simpson scale based on their mean intensity, i.e., the average was taken of 11 the intensity values in the best-track dataset reported within an IOP.

The categories of intensity change or rate of intensification of each IOP were determined by computing the rate of change of intensity 132 of the TC during the IOP. Several definitions exist of intensity change (e.g. Hendricks et al. 2010; Rogers et al. 2013; Cione et al. 2013), yich measure the rate of change in wind speed reported in the best-track dataset in a given period. Here, as in Hendricks $e t$ al. (2010), ve use the following categories of intensity change: intensifying IOPs were defined as $\Delta I / \Delta t \geq 10 \mathrm{kt} 24$-h ${ }^{-1}$, weakening IOPs as

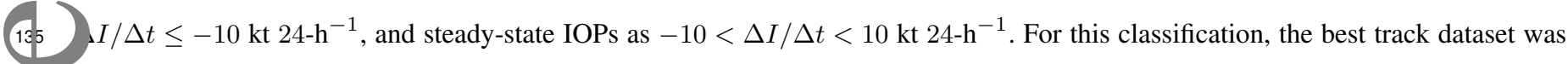
136 rst interpolated to 1 -h resolution. The change in wind intensity $\Delta I$ is computed as the difference between the closest time-step in the interpolated 1-h best track dataset to the middle-point of an IOP, and the intensity found 12-h later. Because this difference is computed roo ove a time window of $12-\mathrm{h}$, the result is multiplied by two to adjust to the criteria above, measured in $\mathrm{kt} 24-\mathrm{h}^{-1}$. This final value is 139 len used to classify the IOPs using the definitions above.

Two alternative definitions of intensity change were employed to evaluate the sensitivity of the results to this definition of intensity change categories. One method computes $\Delta I$ over the subsequent 24 -h period instead of over the following 12 -h. The other definition 142 143 nd end times of the IOP, i.e., this metric aims to measure the change of intensity while measurements were taking place.

Finally, the shape of the resulting gradient wind profile at each height was classified as either peaked or flat by inspecting the fitted wind profile or the derived gradient wind profile as in Kepert (2006a,b) and Schwendike and Kepert (2008). This classification was done using two threshold criteria based on the radial gradient of the wind speed. The first criteria was that peaked profiles have a radial gradient of the wind $|\partial v / \partial r|>1 \mathrm{~m} \mathrm{~s}^{-1} \mathrm{~km}^{-1}$. In this first criteria, stronger storms have a higher likelihood of being classfied as 'peaked', simply because they are stronger and more likely to fit this criteria. Therefore, the second criteria weighs the wind observations by the maximum tangential wind speed $\left(V_{\max }\right)$, as 'peaked' profiles are those that meet the criteria $\partial \frac{V}{V_{\max }} / \partial r>16 \mathrm{~m}{ }^{-1}$. These thresholds aim to capture the strength of the radial decrease in wind speed between the RMW and 2RMW. Composites of wind profiles (see Fig. S1) show that these criteria are able to separate wind profiles where the maximum wind decreases more rapidly (peaked) compared to those with a smoother weakening in wind speed (flat) outside the RMW.

Section 3 will present examples of peaked and flat wind profiles illustrating how these threshold are a reasonable estimation of when a profile is peaked or flat. Table 1 als a presents the mean intensity $\bar{I}(k t)$ of each IOP, the intensity category on the Saffir-Simpson Scale 


\section{2.4. Gradient wind balance}

163 The gradient wind is defined through the balance of the pressure gradient force by the sum of the centrifugal and the Coriolis force 164 (Holton and Hakim 2012), as follows:

$$
\frac{1}{\rho_{0}} \frac{\partial p}{\partial r}=\frac{V_{g}^{2}}{r}+f V_{g}
$$

165 yhere $\rho_{0}$ is the density, $r$ is the distance to storm centre, $\partial p / \partial r$ is the radial pressure gradient, $f$ is the Coriolis parameter, and $V_{g}$ is 166 the gradient wind.

As in many previous studies, the degree of GWB is diagnosed in an axisymmetric framework, therefore, GWB refers here to the 168 balance of forces in the azimuthal mean. This assumption is noteworthy as several factors can induce asymmetry in the wind flow different quadrants of the storm. For instance, an otherwise balanced vortex might have an azimuthal wave-number 1 inertia wave uperimposed, or a motion-induced asymmetry. Hence, due to the limited number of dropsonde observations and the need to account (17. ) $r$ asymmetries, the azimuthal mean assumption is necessary.

172 The degree of GWB is commonly assessed by comparing the observed pressure or wind speeds with those estimated by the gradient wind equation. To better quantify the difference between the observed and estimated winds and possible method biases, the departure gradient wind balance or agradient wind $\left(V_{a g}\right)$ is defined (Kepert 2006a) as the difference between observed azimuthal $u_{\theta}$ and 175 stimated gradient $V_{g}$ winds, i.e.:

$$
V_{a g}=u_{\theta}-V_{g}
$$

176 The agradient wind $\left(V_{a g}\right)$ will be used as a quantitative measure of the degree of GWB.

For the purpose of dignosing gradient wind from dropsonde observations, a continuous function is fitted to pressure or wind 178 bservations (e.g. Kepert 2006a; Schwendike and Kepert 2008; Bell et al. 2012). If pressure observations are fitted then the gradient wind equation (equation 1) needs to be differentiated, whereas if wind observations are fitted, the gradient wind equation will need to be integrated. The fitted continuous function of pressure or wind is then used to solve equation 1 to compute the remaining field, which is considered an estimation of the values of the field if the wind flow was in GWB. For instance, M14 fitted a polynomial (degree 2) function to pressure observations and then estimated the gradient wind $\left(V_{g}\right)$ from the gradient wind equation. Schwendike and Kepert (2008) analysed both pressure-to-wind and wind-to-pressure functions by using a piece-wise function defined by Willoughby et al. (2006), hereafter the WDR function.

The polynomial pressure function $P(r)$ to be fitted, as defined in several studies (e.g. Bell et al. 2012), can be written as:
This article is protected by copyright. All rights reserved. $P(r)=\sum_{k=0}^{n} a_{k} r^{k}$, 
where $n$ is the degree of the polynomial, $a_{k}$ are the coefficients to be determined by the fit, and $r$ is the distance to storm centre. While M14 used $n=2$, Didlake and Houze (2011) used $n=6$ and Bell et al. (2012) $n=3$. Tests using different $n$ values from 2 to 6 showed that increasing $n$ often leads to overfitting and critical poor fits, especially when no dropsondes are found outside the $100 \mathrm{~km}$ radius to contraint the polynomial. For this reason, in this study $n=3$ is used.

WDR showed that a piece-wise function provided more realistic radial pressure gradients and radial wind profiles than previously used profiles (e.g. Holland 1980). The WDR function was defined as a wind function over three regions: the inner, transition, and the outer region. The inner region wind profile $V_{i}(r)$ is defined between the storm centre and the RMW, i.e., in the interval $(0 \leq r \leq R M W) . V_{i}(r)$ is given by the following:

$$
V_{i}(r)=V_{\max }\left(\frac{r}{R M W}\right)^{n}
$$

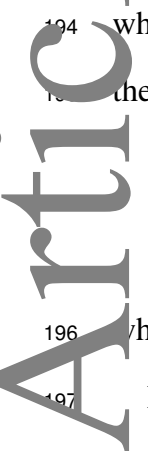

where $V_{\max }$ is the maximum tangential wind and $n$ is an exponent to be fitted to the data. In the outer region, the wind profile $V_{o}(r)$ is he sum of two weighted-functions with different length scales:

$$
V_{o}(r)=V_{\max }\left[(1-A) e^{-\frac{r-R M W}{L_{1}}}+A e^{-\frac{r-R M W}{L_{2}}}\right],
$$

here $L_{1}$ and $L_{2}$ are e-folding distances, and $A$ is a relative weight of $L_{1}$ and $L_{2}$.

Finally, the wind profile in the transition region $V_{t z}$, for $R_{1} \leq r \leq R_{2}$ is a linear combination of equations 4 and 5 , given by:

$$
V_{t z}(r)=V_{i}(1-w)+V_{o} w
$$

${ }^{3}$ vhere $w$ is a polynomial of degree 9. See Kepert (2006a), Willoughby et al. (2006) and Schwendike and Kepert (2008) for further details.

The fit of the WDR function follows closely the method outlined in Kepert (2006a) and Schwendike and Kepert (2008) as the 201 pressure version of the WDR wind fit is found by radially integrating the gradient wind equation using the WDR wind function. - Yowever, there are relevant differences from these previous studies in the fit optimization technique and approach as follows.

The pressure version of the WDR function was fitted to the pressure observations from the dropsonde profiles every 100-m using non-linear squares optimization technique in three steps. The minimum and maximum values of these parameters for this fitting 205 rocedure are shown in Table 2. The first step is to fit the inner region observations to the inner region pressure profile using equation 2064 which results in fitted values of $V_{\max }$ and $R M W$. Using the $V_{\max }$ and $R M W$ from the best fit in the inner region, the outer region observations are fitted to the outer region pressure function to estimate $A, L_{1}$ and $L_{2}$. In short, step 1 and 2 constrain $V_{\max }, R M W$ 208 and $A$. The third and final step then fits the pressure observations simultaneously in the inner, outer and transition regions holding $V_{\max }, R M W$ and $A$ fixed from the values obtained by the previous steps to produce a continous pressure profile. This profile is then iteratively optimized to find the values of $n, L_{1}$ and $L_{2}$ that produce the best fit across all the observations. In other words, the final step optimizes a fit of the optimized individual fits per region using the constraints given by the first two steps. The gradient wind $V_{g}$ is then obtained by numerically solving the gradient wind equation (equation 1) using the fitted pressure profile (either the polynomial or the WDR function) via a numerical quadrature method.

\section{GWB Analysis of Selected IOPs}


225 tangential wind speed.

The first example (IOP 71) falls between the second and third IOPs of M14 and the second example shown here corresponds to the ourth period analysed in M14. Figure 2a, b show the pressure observations and the two continuous pressure profiles for the first case, 228 igure $2 \mathrm{c}, \mathrm{d}$ show the residual, which is the difference between the fits and the observations, and Figure $2 \mathrm{e}$, $\mathrm{f}$ show the gradient wind 220 from both fitting methods. Figure $2 \mathrm{a}, \mathrm{b}$ highlights the differences between the polynomial pressure function and the WDR pressure tonnction, particularly in the shape of the pressure profile. Figure $2 \mathrm{e}$, f show differences in the shape and magnitude of the gradient wind 231 profile. Although the magnitude of the residuals is very similar (Figure $2 \mathrm{c}, \mathrm{d}$ ), the polynomial residuals are mostly positive outside of 232 he $50 \mathrm{~km}$ radius, suggesting that the fit misses the data outside the RMW. The WDR fit does not have this problem. Another relevant difference between the methods is the shape of the fitted pressure profiles which determines the radial gradient of pressure.

234

Since the gradient wind equation refers to the radial gradient of pressure, the resulting gradient wind estimations $V_{g}$ have different hapes and RMW locations. The polynomial $V_{g}$ estimates the RMW to be in the outer region of the storm, far away from the observed MW, whereas the WDR $V_{g}$ produces an RMW very close to the observed value. M14 argued that the misrepresentation of the RMW 237 cation (of the polynomial $V_{g}$ ) was further evidence of the "inaccuracy of the gradient wind for characterizing the structure of the 238 Yortex". However, Figure 2 suggests that some of the results presented by M14 were an artefact of the method and not indicative of the degree of GWB, for instance, the location of the RMW. A qualitative assessment of the polynomial $V_{g}$ suggest that this IOP was rgradient in the inner-core both at the 600-m level and above. The two methods disagree on the degree of GWB as the WDR fit 241 iggests a flow close to balance at both levels in the inner-core.

Figure 3 shows the results for a second example IOP of Hurricane Earl (IOP 75), which can be compared to Figure 11 and 12 of M14. The RMW is located in different regions depending on the fit and the flow near the centre of the storms can be classified (6i) supergradient by the polynomial fit but is closer to balance by the WDR function, as was the case in the previous example. The hean gradient wind anomaly was estimated for each level and weighted by the maximum wind speed to render a percentage value. The reported 32\% supergradient wind by M14 for this period is confirmed and also reduced to less than $10 \%$ in the estimate by the

WDR function. A qualitative assessment of both the WDR and the polynomial $V_{g}$ suggest that this IOP had supergradient winds in the azimuthal mean in the boundary layer, although by noticeable different magnitudes. Very similar features were observed in the analysis of the remaining nine periods of Hurricane Earl (see Table 1). Three of these periods were also analysed by M14, who diagnosed supergradient winds in these periods in the inner-core across the boundary layer but also at the 2000-m level (see their Figure 12).

Hurricane Rita (2005), another well-studied storm, experienced an eyewall replacement cycle that modified the storm structure and intensity (see e.g. Rogers and Uhlhorn 2008; Judt and Chen 2010). Didlake and Houze (2011) and Bell et al. (2012) used the polynomial fit $(n=6)$ to analyze the degree of GWB of Rita using dropsonde observations and concluded that Rita had supergradient winds in the boundary layer. Figure 4 shows the pressure functions, residuals and $V_{g}$ calculations for both the polynomial and WDR functions for IOP 44 (Table 1). As for Hurricane Earl, the residuals from the fits are of similar magnitude, but the residuals for the polynomial fit are mostly positive for radii between 50 and $90 \mathrm{~km}$, whereas the residuals for the WDR fit appear evenly distributed across the abscissa. 

$V_{g}$. In fact, the mean $V_{a g}$ found for the polynomial $V_{g}$ was $+24 \mathrm{~m} \mathrm{~s}^{-1}$ and $+20 \mathrm{~m} \mathrm{~s}^{-1}$ for the 600 and 1800 -m levels, respectively.

The $V_{a g}$ obtained from the WDR $V_{g}$ also shows highly supergradient winds; however, the magnitude of $V_{a g}$ is different from the polynomial results. While some of the resulting $V_{a g}$ from the WDR $V_{g}$ are over $+20 \mathrm{~m} \mathrm{~s}^{-1}$, the mean $V_{a g}$ is $+8 \mathrm{~m} \mathrm{~s}^{-1}$ and $+2 \mathrm{~m} \mathrm{~s}^{-1}$ $268)$ For instance, another well-studied storm, Hurricane Isabel (2003), showed strong boundary layer winds (Montgomery et al. 2006; Bell and Montgomery 2008) and was diagnosed as supergradient in the boundary layer using the WDR fit (Schwendike and Kepert 2008). The $V_{g}$ and $V_{a g}$ from both fits are shown in Figure 5 during 16-21 h UTC on 12 September, 2003 (IOP 20 from Table 1 ). The $V_{a g}$ (Fig. 5c, d) suggests that Isabel had supergradient winds, i.e., several $V_{a g}$ observations of over $+40 \mathrm{~m} \mathrm{~s}-1$, according to the polynomial 269 sig nomalies from the polynomial fit were significantly diffferent to 0 at the $99 \%$ confidence level at both 600 and $1800 \mathrm{~m}$, whereas the (bin 271 anomalies resulting from the WDR fit were only significant at the $600 \mathrm{~m}$ level to the $95 \%$ confidence level.

272 The results of the WDR function support the conclusion of Schwendike and Kepert (2008) that the flow in the upper boundary layer near the RMW was supergradient whereas the flow outside the RMW and above the boundary layer was in balance. This conclusion near t

274 changes depending on the method. While the polynomial fit suggests that the flow is supergradient at all height levels near the RMW, the results from the WDR function show that the supergradient winds were observed only in the upper boundary layer (600 m).

The polynomial fit more frequently diagnosed supergradient winds, i.e., typically larger and positive $V_{a g}$ were diagnosed using the 277 olynomial fit compared to the WDR fit, not just for the cases of Earl and Rita but consistently for most of the analysed IOPs. For 278 Xxample, IOP 13 (see Table 1) of Hurricane Michelle (2001), shown in Figure 6, illustrates the different gradient wind anomalies resulting from each method. Figure $6 \mathrm{a}, \mathrm{b}$ shows the wind observations and gradient wind profiles diagnosed from the polynomial and R fits. The polynomial fit has an overall positive $V_{a g}$ both at the 600 and 1800-m levels, whereas the WDR fit shows a balanced 281 ow in the upper boundary layer and a weakly subgradient flow above (Figs. 6c, d). These differences in the gradient wind anomalies 32 between the methods were determined to be statistically significant at $600 \mathrm{~m}$ at the $95 \%$ confidence level by a Welch t-test, but the differences are not significant at the 1800-m level.

To illustrate the difference between peaked and flat wind profiles and their relevance to determine the degree of GWB, Figure 7 285 hows the wind profiles of IOPs 80, 37 and 31 for Hurricanes Hilary (2011), Frances (2004) and Ivan (2004), respectively. The radial gradient of the wind in and above the boundary layer, classified each of these IOPs in distinct categories as explained in section 2.3 . The WDR wind profile is used to highlight the difference between the wind profiles given this method's superior skill in accurately depicting wind profiles. Hurricane Hilary (Fig. 7a, b) was a storm with a peaked wind profile at the top of the boundary layer and a flat

wind profile above $(1600 \mathrm{~m})$; such cases are referred to as "mixed cases". Hurricanes Frances and Ivan illustrate a flat and a peaked wind profile, respectively, both in and above the boundary layer. The wind profile of Frances (Fig. 7c, d) shows a small decrease in wind speed outside of the RMW, i.e. the radial gradient of wind speed outside the RMW is small compared to the same metric for Ivan. Consider, for example, the wind speeds in Figure $7 \mathrm{c}, \mathrm{d}$ at radii 30 and $75 \mathrm{~km}$ which are $\approx 55$ and $40 \mathrm{~m} \mathrm{~s}^{-1}$, i.e., in a $25 \mathrm{~km}$ radial distance, the wind speed changed by only $\sim 15 \mathrm{~m} \mathrm{~s}^{-1}$. In contrast, a similar measure (wind speed at 40 and $60 \mathrm{~km}$ ) for Ivan (Figure 7e, f) showed a decrease in wind speed of nearly $20 \mathrm{~m} \mathrm{~s}^{-1}$ over a shorter distance. This strong contrast in the radial gradient of the wind speed is the main difference between flat profiles (Hurricane Frances in Fig. 7c, d) and peaked wind profiles (Hurricane Ivan in Figure 7e ,f). Another example of a peaked wind profile was shown in Figure 4e, f. 

312 good assumption for the flow of TCs. Alternatively to PDFs, Figures S2 and S3 show the same distributions in the form of cumulative

This section expands on the previous results by analysing the composites from the 91 IOPs. These composites refer to IOPs separated by i) mean intensity, ii) intensity change rate, and iii) the shape of the wind profile, as explained in the methods section. In addition, the composites were separated by the height of the measurement to analyze whether the degree of GWB changes depending on the vertical layer, for instance, whether supergradient wind is more likely to be diagnosed in the boundary layer than in the free troposphere. Given that the WDR method represents the shape of wind profiles best, results in this section mostly show the $V_{g}$ and the $V_{a g}$ computed from the WDR pressure function. As in the previous sections, the gradient wind anomalies $V_{a g}$ are calculated based on the azimuthal mean wind in an axisymmetric framework.

Probability density functions (PDFs) of the $V_{a g}$ are used to summarize the differences between the composites. PDFs are useful this instance since they convey information on the distribution of the $V_{a g}$ from each composite, thus highlighting the mean and read of the difference between the observed wind and the diagnosed gradient wind. Figure 8 shows the PDFs of the diagnosed $V_{a g}$ composited based on four characteristics: a) intensity, b) rate of intensification, c) shape of wind profile, and d) height. Similarly, Table reports the mean and standard deviation of each composite, as well as the likelihood of showing markedly supergradient and markedly subgradient flow. These probabilities were estimated by integrating the density function using a trapezoid numerical method. The mean of all the diagnosed $V_{a g}$ for all heights is $1 \mathrm{~m} \mathrm{~s}^{-1}$ (Table 3), which confirms the results of Willoughby (1990) that GWB is generally a 313 censity functions and boxplots, respectively, both of which agree well with the PDF analysis that follows.

314 The PDF of all of the observations in the inner-core $(R M W / 2<r<3 R M W / 2)$, i.e., 16,000 dropsondes observations, is labelled fontrol to illustrate the distribution of the gradient wind anomalies $V_{a g}$ of the 91 IOPs. This distribution shows that gradient wind T10 nomalies are roughly evenly distributed across negative and positive anomalies. The Control PDF is relatively broad because this 317 sample contains observations from multiple height levels, different storms sizes and intensities, all of which may produce a number of $\sqrt{310}$ positive and negative gradient wind anomalies. Additionally, this PDF is evenly distributed due to observational errors, turbulence and factors that affect the dropsonde measurements, as well as possible storm asymmetries between storm quadrants.

Figure 8 a indicates that stronger storms have a higher probability of experiencing supergradient flow. The PDFs of the Cat. 3,4 and 5 TCs suggest a more frequent occurrence of $V_{a g}>10 \mathrm{~m} \mathrm{~s}^{-1}$, when compared to the Control and to weaker storms. Table 3 shows hat the stronger the tangential wind in an IOP the higher the likelihood of finding markedly supergradient winds in the inner-core. The 323 arrowest distribution of all composites is from the weakest composite, i.e., the Tropical Storms and Tropical Depressions (TS/TD), 324 hich is also the composite with the lowest standard deviation (Table 3). A narrower distribution is interpreted as being more frequently in balance.

The PDF of the TS/TD category shows the highest probability of gradient wind anomalies in the $0-5 \mathrm{~m} \mathrm{~s}^{-1}$ interval, which suggests a balanced flow with a tendency towards very frequent small positive gradient wind anomalies. This relatively narrower PDF is due to smaller wind speeds in IOPs in the TS/TD category $\left(17-32 \mathrm{~m} \mathrm{~s}^{-1}\right)$, which are usually observed with weaker radial pressure gradients and therefore weaker diagnosed gradient winds compared to stronger storms. Since both the observed and gradient winds are small, the resulting gradient wind anomaly is frequently also small.

The inner-core observations were composited based on the observed rate of intensification as explained in section 2.3 , into steady-state, weakening and intensifying IOPs, shown as PDFs in Fig. 8b. The major difference amongst these categories is that the weakening composite shows a higher likelihood of modest supergradient winds than steady-state and intensifying IOPs. Table 3 shows quantitatively that the highest probability of supergradient flow $\mathrm{p}\left(V_{a g}>15 \mathrm{~m} \mathrm{~s}^{-1}\right)$ is that of the weakening composite. The other difference is that the PDF of the steady-state composite has a higher probability of diagnosing weakly subgradient flow than in 

greatest probability of supergradient flow ( $\left.V_{a g}>10 \mathrm{~m} \mathrm{~s}^{-1}\right)$ is found for observations in the upper-boundary layer (400-600 m) whereas 346 observations close to the surface $(<400 \mathrm{~m})$ show a higher probability of having subgradient flow $V_{a g}<-10 \mathrm{~m} \mathrm{~s}^{-1}$, consistent with (3) e effects surface friction. Gradient wind anomalies above $1500 \mathrm{~m}$ appear to have a higher likelihood of presenting weak subgradient - fow than at other heights and than the Control.

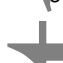

Figure 9 shows the distributions of gradient wind anomalies further divided by both the height and radial location of the dropsonde 350 observation. First, Figure 9a shows that observations in the upper boundary layer near and inside the RMW have a higher likelihood of ${ }_{352}$ being supergradient than observations outside the RMW. The PDF of observations outside the RMW is also narrower than the Control ggesting that the wind is more likely to be balanced in this region.

Second, Figure 9b, c show the PDFs from observations in the inner-core of strong Cat. 4-5 (Fig. 9b) and weak Cat. 1-2 IOPs (Fig.

c) at two different height levels: the upper boundary layer (400-600 m) and above $1500 \mathrm{~m}$. These PDFs show that the degree of GWB F varies depending on both the intensity of the IOP and the height of the measurement. These PDFs are compared against observations from the Control PDF subdivided into two PDFs according to the height level: 400-600 and above $1500 \mathrm{~m}$. Strong storms have a 357 higher likelihood of supergradient wind in the upper boundary layer than above and a higher likelihood of supergradient winds than the 258 Control at the same height. Weak storms also show more frequently supergradient winds in upper boundary layer than above $1500 \mathrm{~m}$, 9 'owever, the likelihood of markedly supergradient winds in Cat. 1-2 IOPs in the upper boundary layer is smaller than that of the Control 360 and of stronger storms at the same height. The PDF of the Cat. 1-2 IOPs at the upper boundary layer shows a bimodal distribution with (361 No peaks: a peak in the very weak subgradient region and another peak showing increased probability of modest supergradient flow (6) $\left.5<V_{a g}<12 \mathrm{~m} \mathrm{~s}^{-1}\right)$.

Furthermore, Figure 9d-f show the observations subsampled by rate of intensification and height. The weakening and intensifying composites show a higher likelihood of markedly supergradient winds in the upper boundary layer than above $1500 \mathrm{~m}$ (Figs. 9d, e).

365

In contrast, the steady-state composite shows a slightly narrower PDF in the upper boundary layer than above $1500 \mathrm{~m}$, suggesting a slightly lower probability of supergradient winds in the upper boundary layer than above. The weakening composite shows a slightly higher probability of markedly supergradient flow $\left(V_{a g}>15 \mathrm{~m} \mathrm{~s}^{-1}\right)$ than the Control and the intensifying PDFs in the upper boundary layer. The steady-state PDF in the boundary layer top also shows a bimodal structure in the same regions as the Cat. 1-2 PDF of Fig. $9 \mathrm{c}$.

Table 3 presents the results of a Welch t-test between each composite and the Control sample, indicating significance at the $95 \%$ and $99 \%$ levels. Moreover, to statistically test the differences between each PDF, a Kolmogorov-Smirnov (KS) test was used, which measures how likely is a distribution, or a PDF, to be simply a sub-sample of another distribution. In other words, this test evaluates whether a given composite is statistically are distinct from all the observations. Both tests show that the rate of intensity change has the

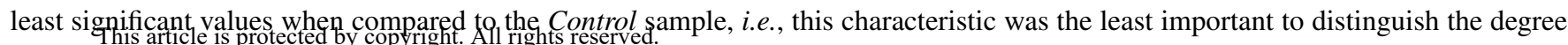



percentile of the distributions is $28 \mathrm{~m} \mathrm{~s}^{-1}$ for the WDR fit and $52 \mathrm{~m} \mathrm{~s}^{-1}$ for the polynomial fit. This behaviour is much less apparent n the left-hand tail, where the distributions are more similar, thus confirming a positive bias of the polynomial fit when compared to ne WDR functions. The more frequent supergradient winds estimated by the polynomial fit confirm the results of the previous section,

hich suggested that the polynomial fit produced stronger supergradient features when compared to the WDR function.

To test the sensitivity of the results to the track dataset used, $V_{a g}$ was computed using two alternative track datasets (best-track and PF) for ten IOPs $(6,20,21,22,39,40,41,42,43$ and 56). The observations resulting from these alternative tracks were analysed sing the two fitting functions as for the WC track. The results of each track for each fitting function were compared in different radial egions: inside, near and outside the RMW. Boxplots (Figure 11) show that the three datasets produce a very similar mean $V_{a g}$. The TPF technique produces a larger spread in $V_{a g}$, particularly near the RMW. However, the impact of the fitting method on the diagnosed 392 gradient wind anomalies $V_{a g}$ is stronger than the track dataset used to locate the dropsondes in a storm-relative framework. The mean $Y_{a g}$ using the polynomial fit (Figure 11b) is higher than the results for the WDR function (Figure 11a), regardless of the track method nd especially near the RMW. The PDFs calculated from the two alternative tracks did not show substantial differences. In particular, (4) mean and spread of each distribution was not significantly different between the track algorithms.

Similarly, to analyze whether the definition of intensity change affected the findings of this section, two alternative definitions of tensity change were implemented (see method section). One definition is based on the change in wind speed in a 24-h period and ther definition considers the difference in intensity during the course of the IOP. The PDFs of the intensity change composites ing the two alternative definitions of intensity change (not shown) were consistent with the results from the first definition, shown in so Figures 8 and 9, i.e., no difference between the intensifying, weakening or steady-state composites. The analysis of the PDFs and the Welch and Kolmogorov-Smirnov statistical tests showed no sensitivity to the definition of intensity change category.

\section{Summary and discussion}

Observations of supergradient winds in TCs have been documented in several case studies, but the question remains as to whether

the degree of GWB is consistently linked to some characteristics of a TC such as intensity. This study expands on previous work by diagnosing the degree of GWB in a sample of 91 IOPs corresponding to 30 TCs in the period of 1999-2012.

Previous studies have used two different functions to fit pressure observations and then diagnose GWB, some studies use the WDR piece-wise function and others use the polynomial function. The two fitting methods were compared for several cases, first by evaluating the skill of the fit in reproducing the main features of the radial pressure gradient and, second, by comparing the diagnosed gradient wind from both methods to the observed tangential wind. This analysis showed that the diagnosed gradient wind profiles were notably different between the polynomial and the WDR functions. Several characteristics of the diagnosed gradient wind profile were sensitive to the fitting function such as the location of the RMW and the maximum wind speed. For example, in two IOPs of Hurricane Earl and one ThOP of Hurricane Rita the polynomial function resulted in mostly positive residuals in the pressure fit outside $50 \mathrm{~km}$ radius, a 

The degree of GWB, quantitatively measured as the difference between the observed winds and the estimated gradient winds, could iffer between using a polynomial and a piece-wise fitting function by more than $40 \mathrm{~m} \mathrm{~s}^{-1}$ for individual observations and a mean nomly difference between the methods of $20 \mathrm{~m} \mathrm{~s}^{-1}$ for some storms.

(424) The polynomial fit frequently diagnosed supergradient winds in the upper-boundary layer and above in other storms, such as 425 Hurricanes Michelle and Rita, which agrees well with previous analyses using the polynomial fit (Bell and Montgomery 2008; Rogers Ind Uhlhorn 2008; Rogers et al. 2015). Above the boundary layer, the polynomial fit also consistently suggests supergradient winds 127 (Fig. 10) whereas the WDR more frequently diagnoses a flow closer to balance (observed as a narrower PDF). Also, the RMW 428 iagnosed from the gradient wind resulting from the polynomial fit occurs at larger radii than observed whereas the WDR fit usually finds a RMW much closer to the observed RMW.

430

These differences amongst methods reinforce the conclusion that the diagnosis of GWB in observations is highly sensitive to the fechnique, in this case the function used for the fit to pressure observations (Gray and Shea 1973; Willoughby 1990; Gray 1991). Given ne better performance of the WDR function in representing observed wind profiles (Willoughby et al. 2006), arguably, the results 433 om the WDR function are more representative than those of the polynomial fit. For instance, the results from the WDR profile are 1434 consistent with our theoretical expectation that the azimuthal mean flow above the boundary layer should usually be relatively close to GWB.

dditionally, this study investigated whether the degree of GWB was statistically linked to mean intensity and intensity change 437 the of the IOP, the height and distance from storm centre of the measurement and the shape of the wind profile at each height level 38 by compositing 91 observation periods using the results from the WDR fit. Figure 12 summarises the results of the PDF analysis by howing the probabilities of finding markedly supergradient and subgradient flows in each of the different composities of intensity, 440 te of intensification, shape of wind profile, etc. The PDF analysis showed that the composite of periods of stronger storms (Cat. 3, 4 441 nd 5) had a significantly higher likelihood of exhibiting supergradient winds than the composites of weaker storms (TS/TD and Cat. 1-2). These differences were accentuated when observations were composited based on both intensity of the IOP and height of the 443 measurement as more supergradient winds are found in the upper boundary layer than above.

The degree of GWB is less related to the rate of intensification. The distributions of the weakening, intensifying and steadystate composites were not significantly different. Weakening storms show a higher likelihood (Fig. 12) of supergradient winds than intensifying storms, particularly in the boundary layer. This result seems inconsistent with the implications of the boundary layer spinup paradigm (Montgomery et al. 2014; Smith and Montgomery 2015; Montgomery and Smith 2017), which argues that supergradient winds in the boundary layer play an important role for intensification.

In contrast, one of the compositing characteristics that led to the largest differences in the PDFs of gradient wind anomalies was the shape of the wind profile, as separating the wind profiles into peaked and flat resulted in significantly different PDFs of gradient wind anomalies. In particular, peaked wind profiles had a higher probability of having supergradient winds above $15 \mathrm{~m} \mathrm{~s}^{-1}$ than periods with a fat wind profile (Fig. 12 ). These results agree with the model of Kepert and Wang (2001), in which the radial distribution of 


\section{^cknowledgement}

- ILGF was supported by a CONACYT-SENER Scholarship (CVU: 701275) and an Oxford-Richards Scholarship. The authors thank Vill Torgerson for his help with the calculation of the track using the translating fitting pressure method. The authors also thank Jeff Kepert for his valuable comments and the discussions on early versions of this manuscript. This and many other studies could not have 467 een done without the NOAA, NASA and USAF teams that have been dedicated to deploy dropsondes for over 20 years. The authors would also like to thank two anonymous reviewers for their constructive comments that have greatly improved this manuscript.

\section{Supporting information}

- Figure S1: Gradient wind profile composites based on shape of wind profile criteria.

- Figure S2: Cumulative density function version of Figure 8.

- Figure S3: Boxplots version of Figure 8.

- Figure S4: PDFs as in Figure 8c, showing the sensitivity to the criteria of peaked and flat profiles.

\section{KeIt rences}

Abarca SF, Montgomery MT, Braun SA, Dunion J. 2016. On the Secondary Eyewall Formation of Hurricane Edouard (2014). Monthly Weather Review 144: 476 3321-3331, doi:10.1175/MWR-D-15-0421.1.

Bell MM, Montgomery MT. 2008. Observed structure, evolution, and potential intensity of category 5 Hurricane Isabel (2003) from 12 to 14 September. Monthly

Weather Review 136: 2023-2046.

479 'ell MM, Montgomery MT, Lee WC. 2012. An axisymmetric view of concentric eyewall evolution in Hurricane Rita (2005). Journal of the Atmospheric Sciences 69: 2414-2432

481 raun SA, Kakar R, Zipser E, Heymsfield G, Albers C, Brown S, Durden SL, Guimond S, Halverson J, Heymsfield A, Ismail S, Lambrigtsen B, Miller T, Tanelli S, Thomas J, Zawislak J. 2013. NASA's Genesis and Rapid Intensification Processes (GRIP) field experiment. Bulletin of the American Meteorological Society 94: $345-363$. 
Atmospheric Sciences 67: 3581-3599.

Kepert JD. 2001. The dynamics of boundary layer jets within the tropical cyclone core. Part I: Linear theory. Journal of the Atmospheric Sciences 58: $2469-2484$.

505 Kepert JD. 2005. Objective analysis of tropical cyclone location and motion from high-density observations. Monthly Weather Review 133: $2406-2421$.

506 epert JD. 2006a. Observed boundary layer wind structure and balance in the hurricane core. Part I: Hurricane Georges. Journal of the Atmospheric Sciences

507 63: 2169-2193.

508 Yepert JD. 2006b. Observed boundary layer wind structure and balance in the hurricane core. Part II: Hurricane Mitch. Journal of the Atmospheric Sciences 63: 2194-2211.

510 Kepert JD, Wang Y. 2001. The dynamics of boundary layer jets within the tropical cyclone core. Part II: Nonlinear enhancement. Journal of the Atmospheric Sciences 58: 2485-2501.

Landsea CW, Franklin JL. 2013. Atlantic hurricane database uncertainty and presentation of a new database format. Monthly Weather Review 141: $3576-3592$.

513 Miyamoto Y, Satoh M, Tomita H, Oouchi K, Yamada Y, Kodama C, Kinter James I. 2014. Gradient Wind Balance in Tropical Cyclones in High-Resolution 514 Global Experiments. Monthly Weather Review 142(5): 1908-1926, doi:10.1175/MWR-D-13-00115.1.

515 Iontgomery MT, Bell MM, Aberson SD, Black ML. 2006. Hurricane Isabel (2003): New insights into the physics of intense storms. Part I: Mean vortex 56 structure and maximum intensity estimates. Bulletin of the American Meteorological Society 87: $1335-1348$.

Montgomery MT, Smith RK. 2017. Recent developments in the fluid dynamics of tropical cyclones. Annual Review of Fluid Mechanics 49: 541-574.

Aontgomery MT, Zhang JA, Smith RK. 2014. An analysis of the observed low level structure of rapidly intensifying and mature hurricane Earl (2010). Quarterly Journal of the Royal Meteorological Society 140: 2132-2146.

rgrass AG, Willoughby HE. 2009. Diabatically induced secondary flows in tropical cyclones. Part I: Quasi-steady forcing. Monthly Weather Review 137: $805-821$.

Rogers RF, Reasor PD, Lorsolo S. 2013. Airborne doppler observations of the inner-core structural differences between intensifying and steady-state tropical cyclones. Monthly Weather Review 141: 2970-2991.

ogers RF, Reasor PD, Zhang JA. 2015. Multiscale structure and evolution of hurricane earl (2010) during rapid intensification. Monthly Weather Review 143: $536-562$.

ogers RF, Uhlhorn E. 2008. Observations of the structure and evolution of surface and flight-level wind asymmetries in Hurricane Rita (2005). Geophysical Research Letters 35, doi:10.1029/2008GL034774.

528 Sanger NT, Montgomery MT, Smith RK, Bell MM. 2014. An observational study of tropical cyclone spinup in Supertyphoon Jangmi (2008) from 24 to 27 529 September. Monthly Weather Review 142: 3-28.

530 Schwendike J, Kepert JD. 2008. The boundary layer winds in Hurricanes Danielle (1998) and Isabel (2003). Monthly Weather Review 136: $3168-3192$.

Shapiro LJ, Willoughby HE. 1982. The response of balanced hurricanes to local sources of heat and momentum. Journal of the Atmospheric Sciences 39: $378-394$.

Smith RK, Montgomery MT. 2010. Hurricane boundary-layer theory. Quarterly Journal of the Royal Meteorological Society 136: $1665-1670$.

Smith RK, Montgomery MT. 2015. Toward Clarity on Understanding Tropical Cyclone Intensification. Journal of the Atmospheric Sciences 72: 3020-3031.

Smith RK, Montgomery MT. 2016. Understanding hurricanes. Weather 71: 219-223.

Stern DP, Kepert JD, Bryan GH, Doyle JD. 2020. Understanding atypical midlevel wind speed maxima in hurricane eyewalls. Journal of the Atmospheric Sciences 77: 1531-1557. 
Vigh J, et al. 2015. FLIGHT+: The extended flight level dataset for tropical cyclones. Tropical Cyclone Data Project, National Center for Atmospheric Research, Research Applications Laboratory, Boulder, Colorado. [Available online at: http://dx. doi. org/10. 5065/D6WS8R93] Accessed: March 25th, 2018.

Wang JJ, Young K, Hock T, Lauritsen D, Behringer D, Black M, Black PG, Franklin J, Halverson J, Molinari J, Nguyen L, Reale T, Smith J, Sun B, Wang Q, Zhang JA. 2015. A long-term, high-quality, high-vertical-resolution gps dropsonde dataset for hurricane and other studies. Bulletin of the American Meteorological Society 96: 961-973.

Willoughby H. 1979. Forced secondary circulations in hurricanes. Journal of Geophysical Research: Oceans 84: $3173-3183$.

Willoughby HE. 1990. Gradient balance in tropical cyclonesff. Journal of the Atmospheric Sciences 47: 265-274.

Willoughby HE, Clos JA, Shoreibah MG. 1982. Concentric eye walls, secondary wind maxima, and the evolution of the hurricane vortex. Journal of the Atmospheric Sciences 39: 395-411.

Willoughby HE, Darling RWR, Rahn ME. 2006. Parametric representation of the primary hurricane vortex. Part II: A new family of sectionally continuous profiles. Monthly Weather Review 134: 1102-1120.

Zhang JA, Rogers RF, Nolan DS, Jr FDM. 2011. On the characteristic height scales of the hurricane boundary layer. Monthly Weather Review 139: 2523-2535. 550 Zhang JA, Rogers RF, Reasor PD, Uhlhorn EW, Marks Jr FD. 2013. Asymmetric hurricane boundary layer structure from dropsonde composites in relation to 551 the environmental vertical wind shear. Monthly Weather Review 141: 3968-3984.
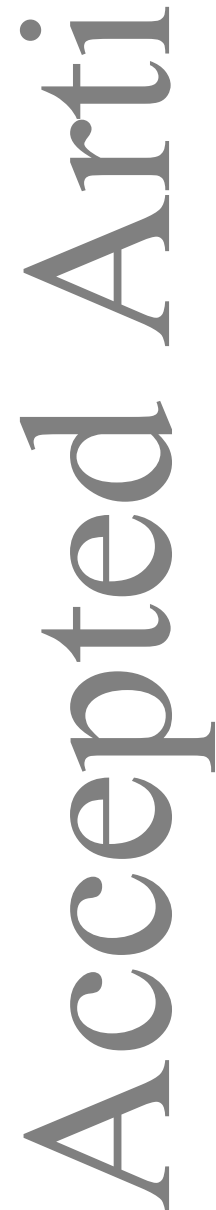
a)

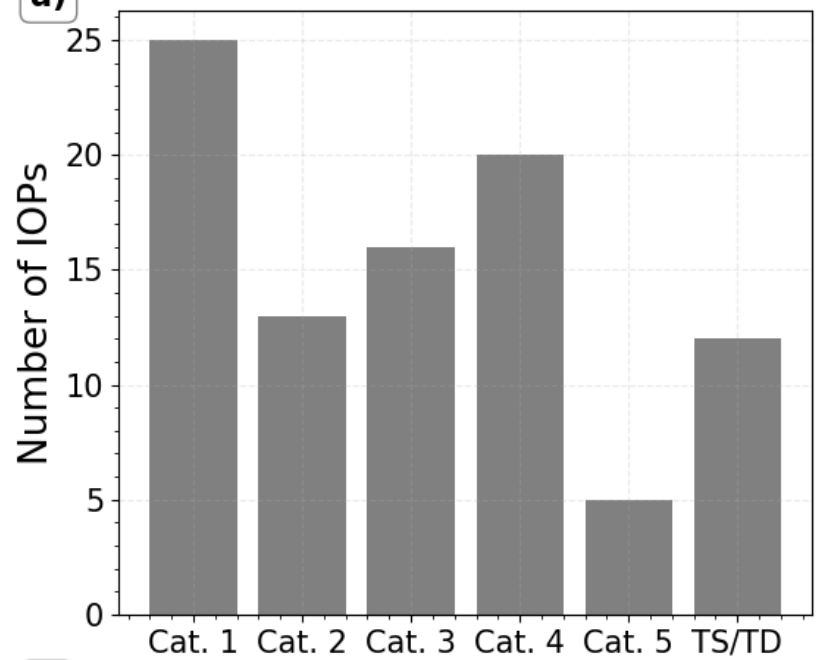

c)

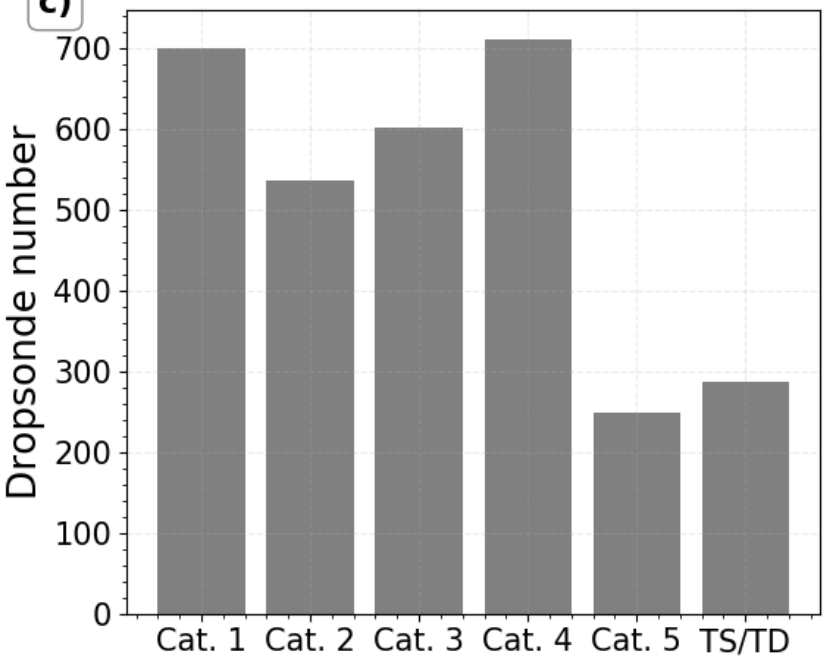

b)

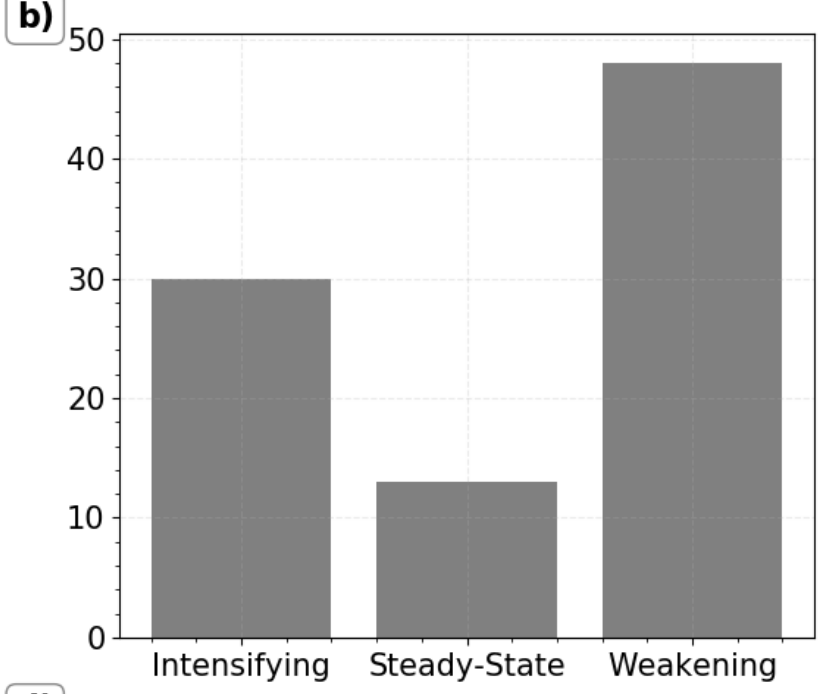

d)

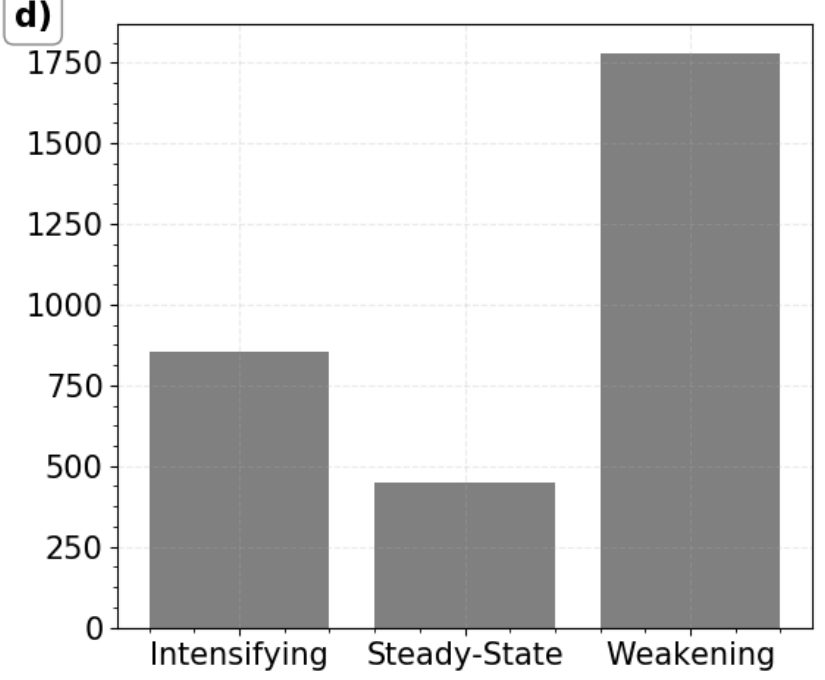

igure 1. (a, b) Number of IOPs and (c, d) total dropsonde number for each of the (a, c) intensity and (b, d) intensity change categories. The total dropsonde number includes only dropsondes within a $220-\mathrm{km}$ radius from the storm centre.

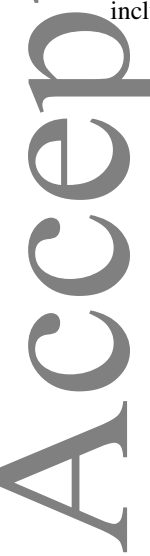



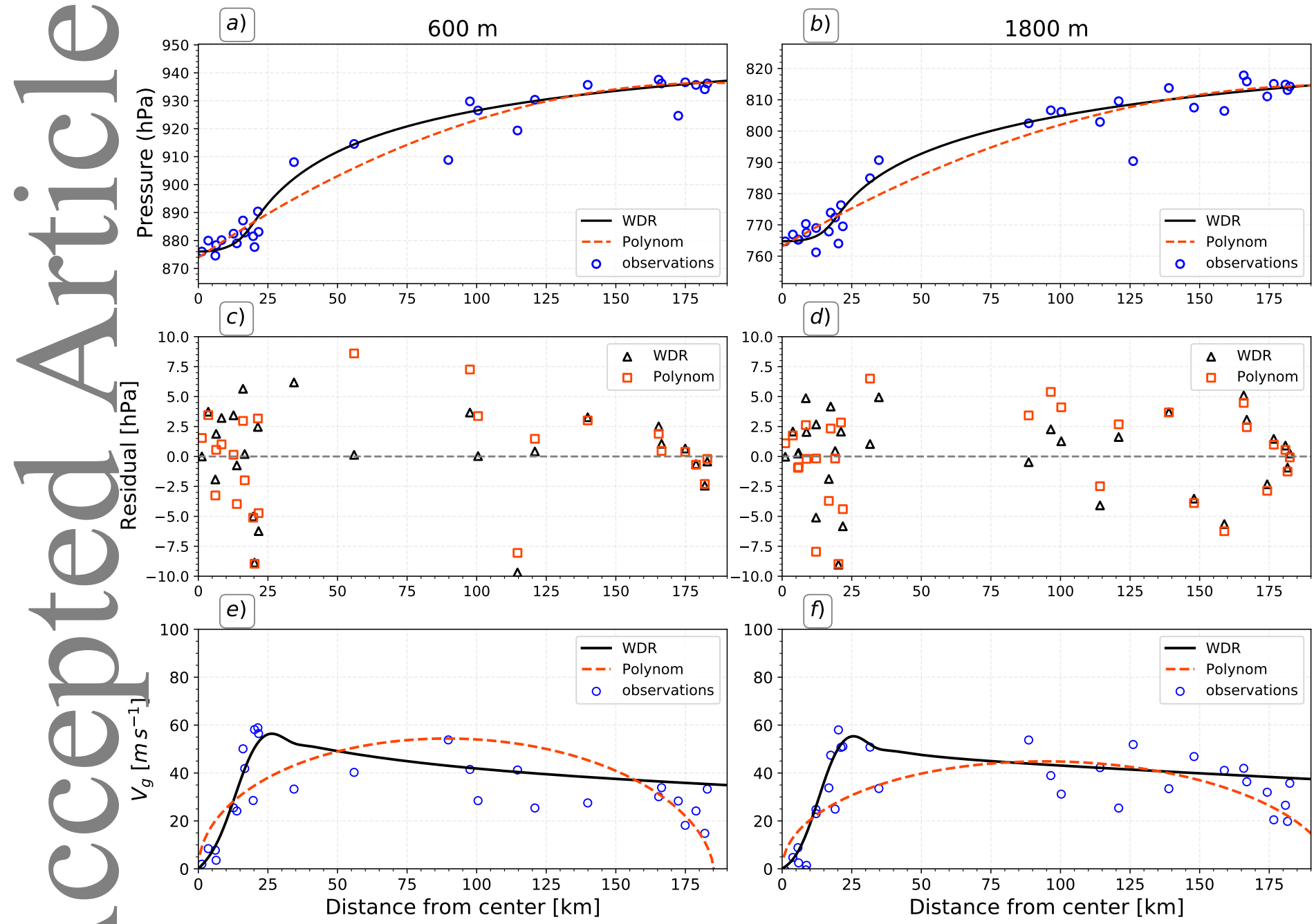

Figure 2. Gradient wind balance assesment during 18 UTC on 30 August to 00 UTC on 31 August 2010 (IOP 72 in Table 1) of Hurricane Earl (2010). (a, b) show the ressure observations (circles) with the polynomial (dashed red) and WDR (solid black) pressure fits. (c, d) show the residuals from the fits, in units of hPa. (e, f) The gradient wind profiles $\left(V_{g}\right)$ from each fitting method is shown, as well as the observed tangential wind (circles). Results are shown at (a, c, e) $600 \mathrm{~m}$ and (b, d, f) $1800 \mathrm{~m}$ height. 
a)

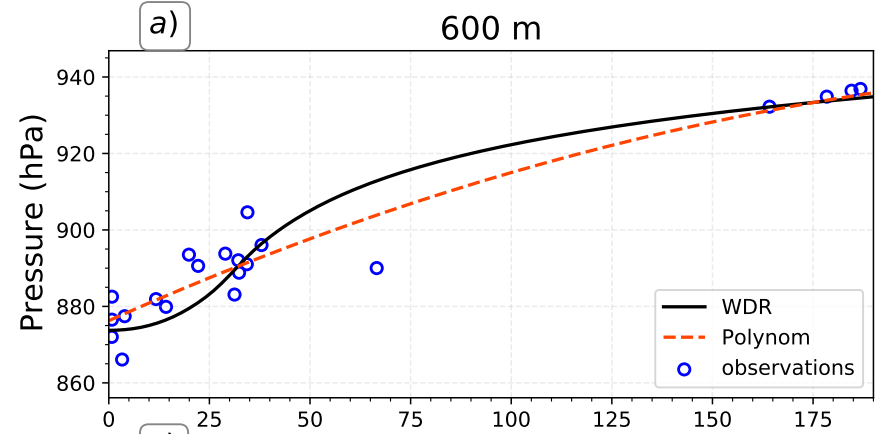

c)

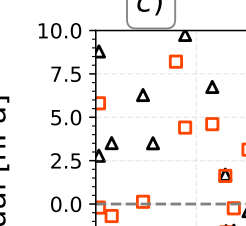

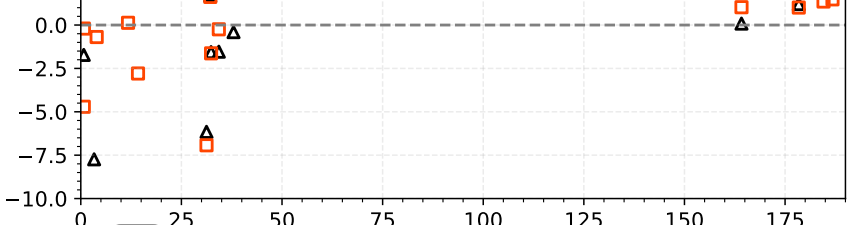

e)

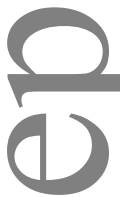

() $\varepsilon$

() 20

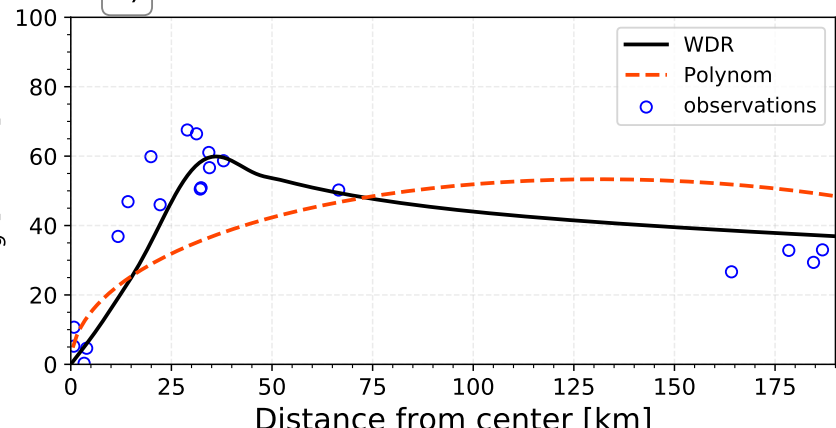

b)

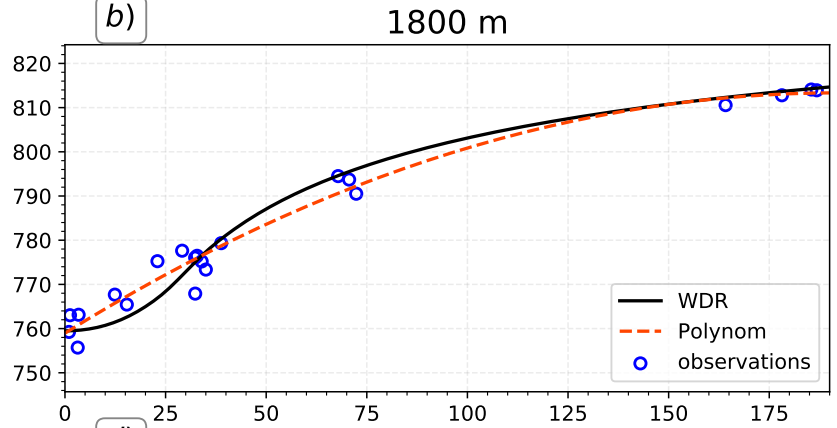

d)
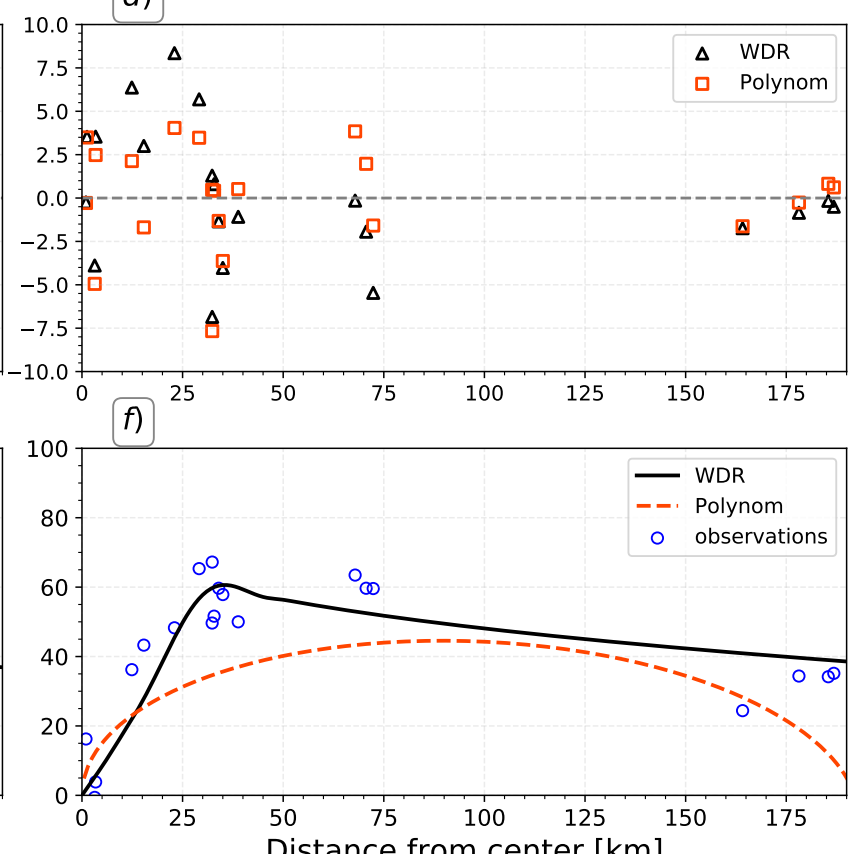

Figure 3. As in Figure 2, but for 06-18 UTC on 2 September 2010 (IOP 75 in Table 1) of Hurricane Earl (2010). 
a)

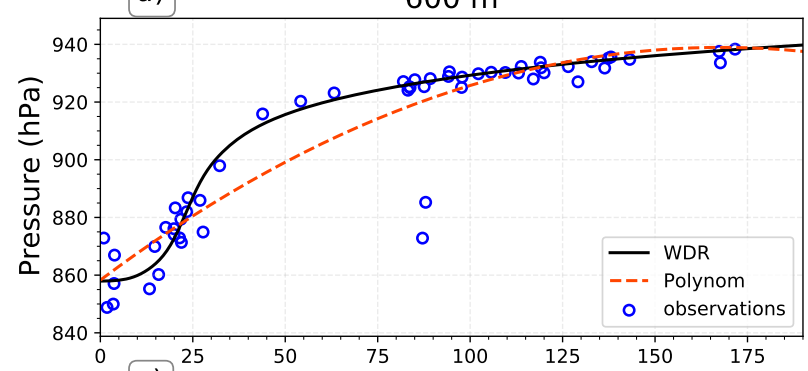

c)

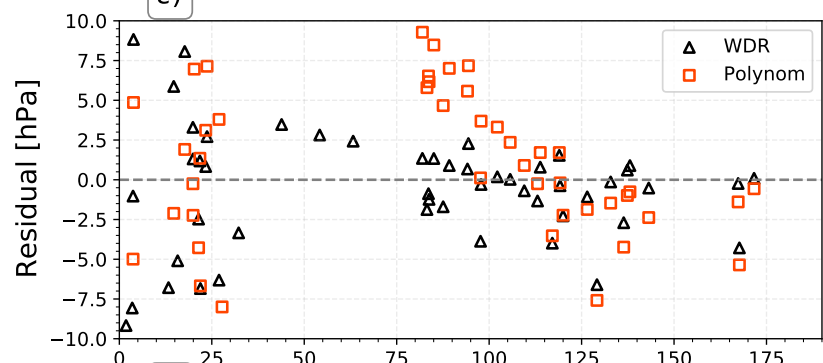

e)

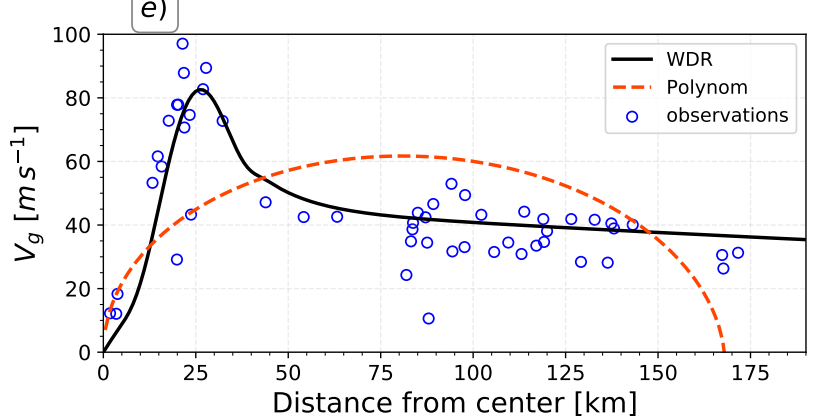

b)

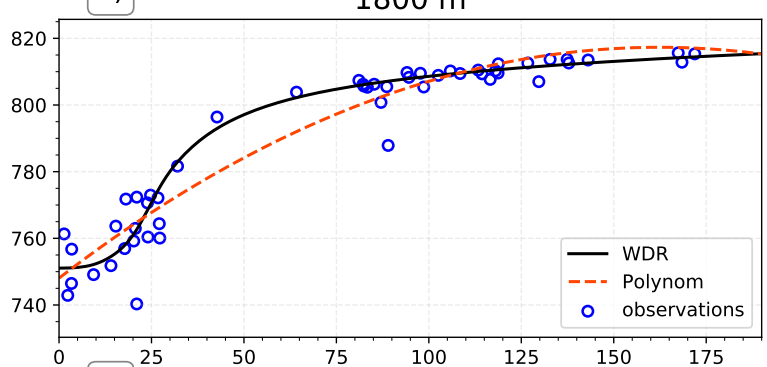

d)
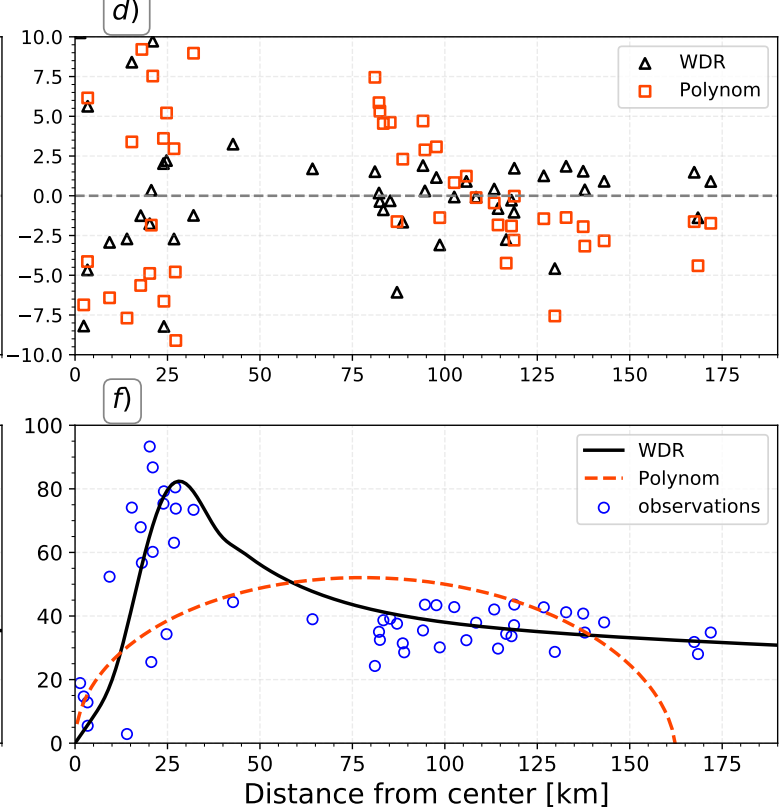

Figure 4. As in Figure 2, but for 15-21 UTC on 21 September, 2005 (IOP 44 in Table 1) of Hurricane Rita (2005). 
a)

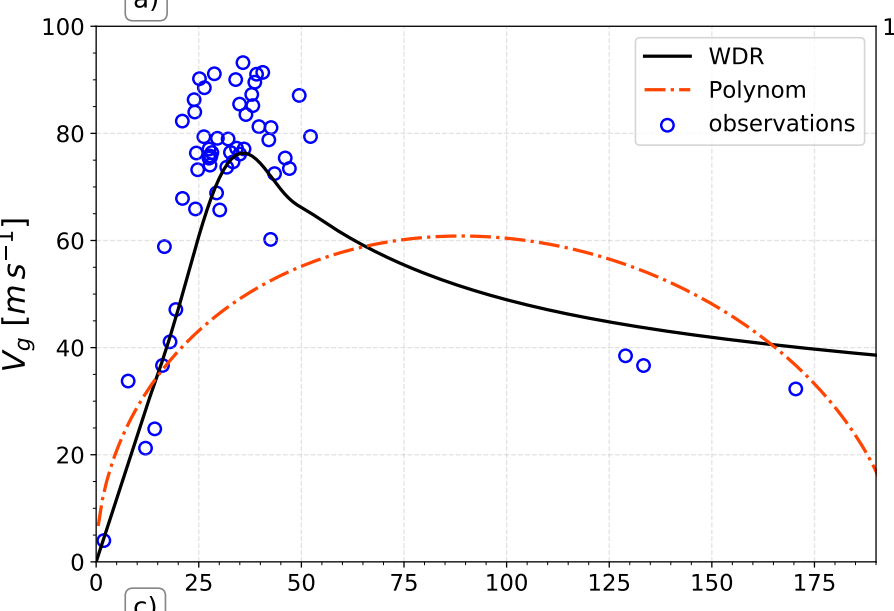

c)

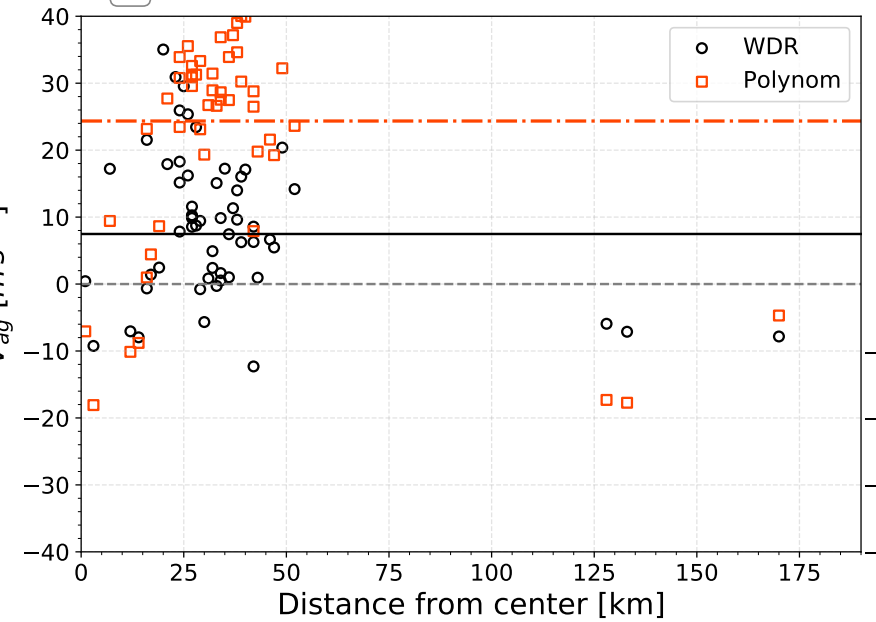

b)

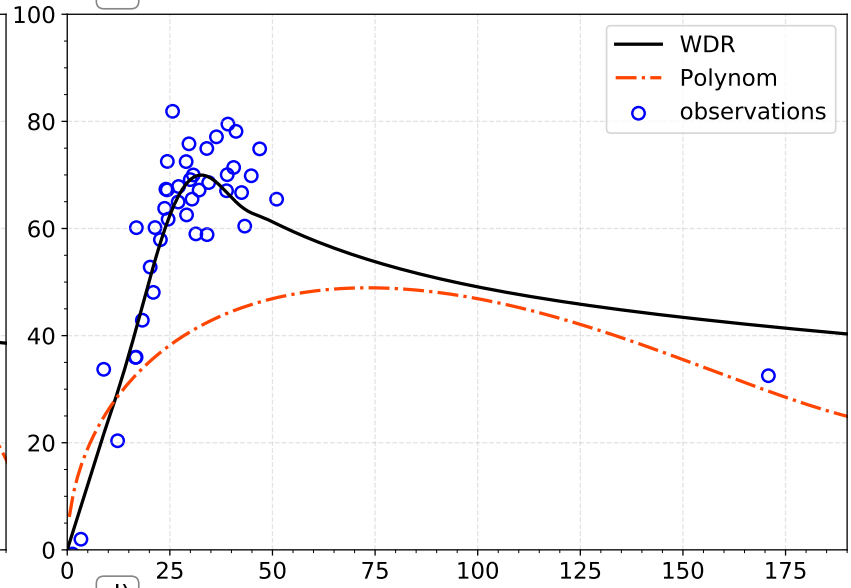

d)

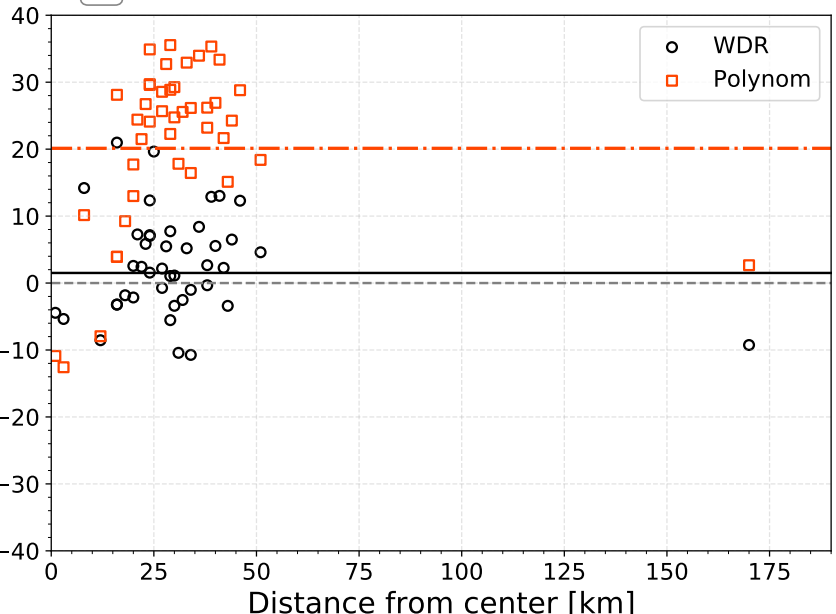

Gigure 5. (a, b) Tangential wind observations (blue circles) and estimated gradient winds ( $V_{g}$ ) from the polynomial (red) and the WDR functions (black) for Hurricane abel for 16-21 UTC on 12 September 2005 (IOP 20 from Table 1) is shown. (c, d) The agradient wind ( $V_{a g}$ ), a measure of the degree of GWB, is shown for each wind bservation. The black (red) dashed line illustrates the mean $V_{a g}$ for the WDR (polynomial) fit. Results are shown at the (a, c) 600-m level and (b, d) the 1800-m level. 
a)

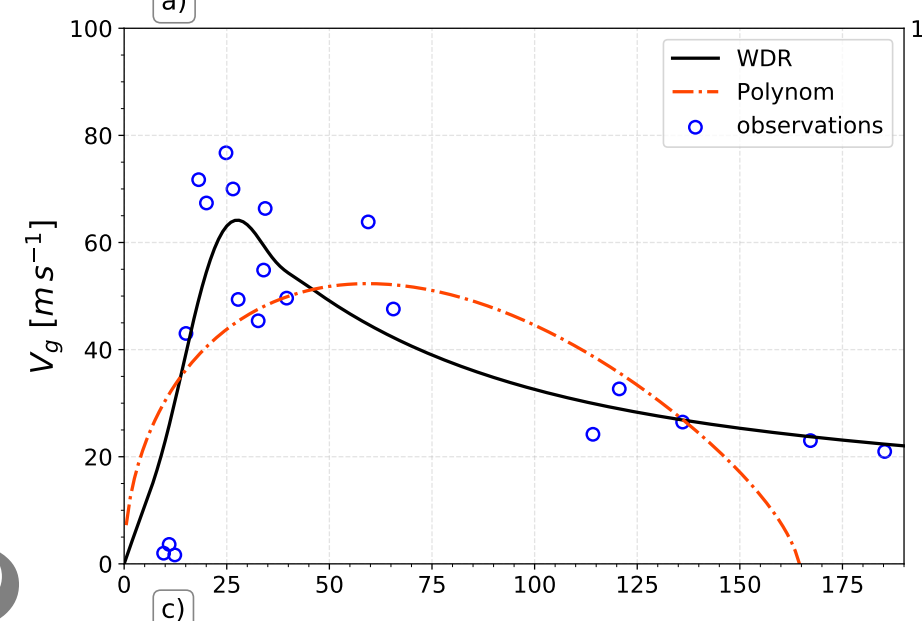

C) 25

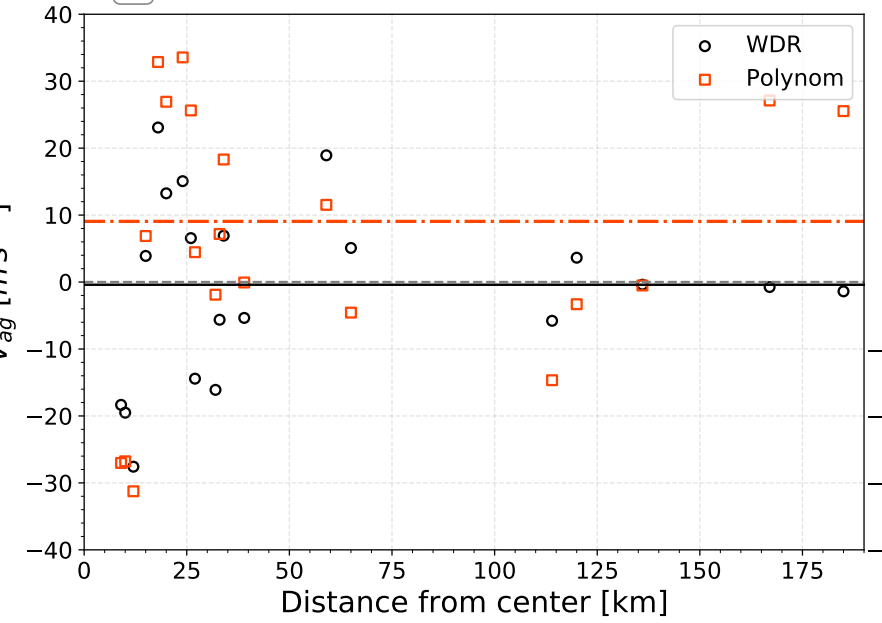

b)

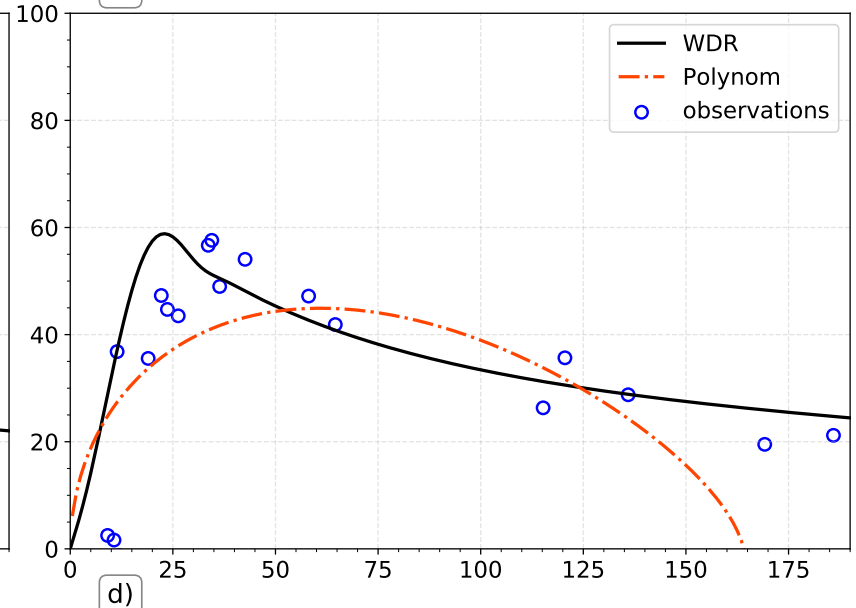

d)

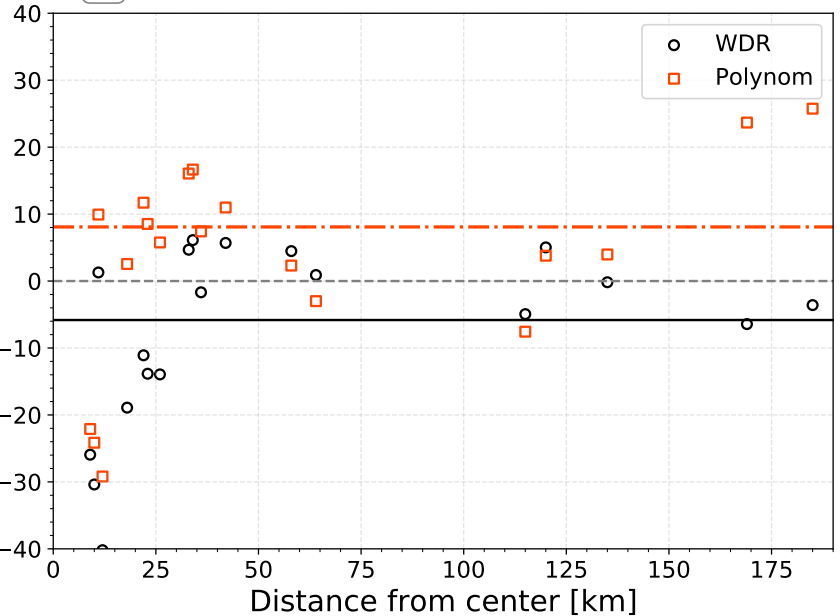

Figure 6. As in Figure 5, but for Hurricane Michelle from 18 UTC 3 November to 05 UTC 4 November (2001) (IOP 13 in Table 1). 

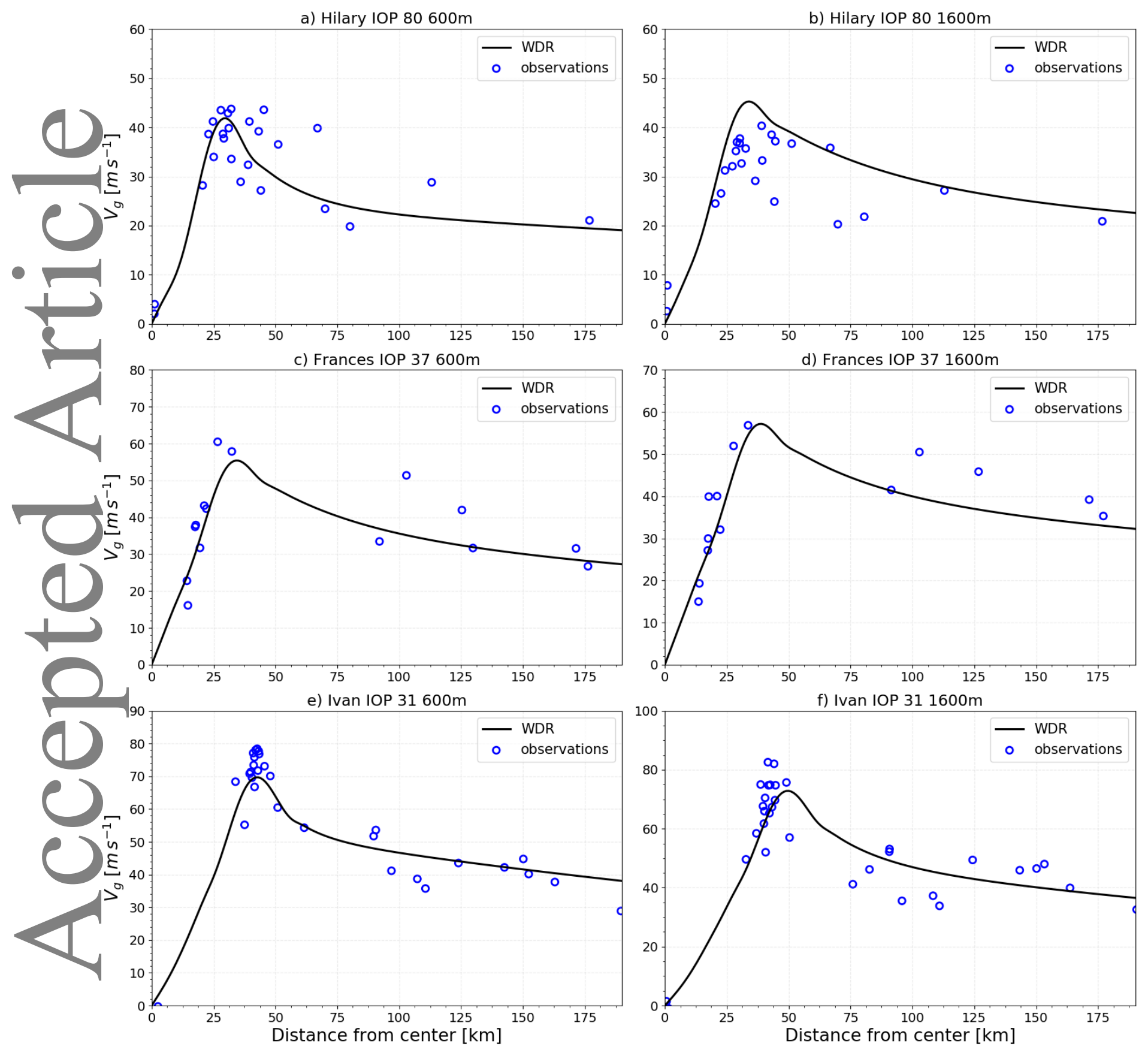

Figure 7. Gradient wind from the WDR function (black) and dropsonde observations (blue circles) for IOPs of Hurricanes (a, b) Hilary (2011), (c, d) Frances (2004), and (e, f) Ivan (2004). Panels (a,c,e) represent the top of the boundary layer $(600 \mathrm{~m})$ whereas (b, d, f) are above the inflow layer (1600 m). 

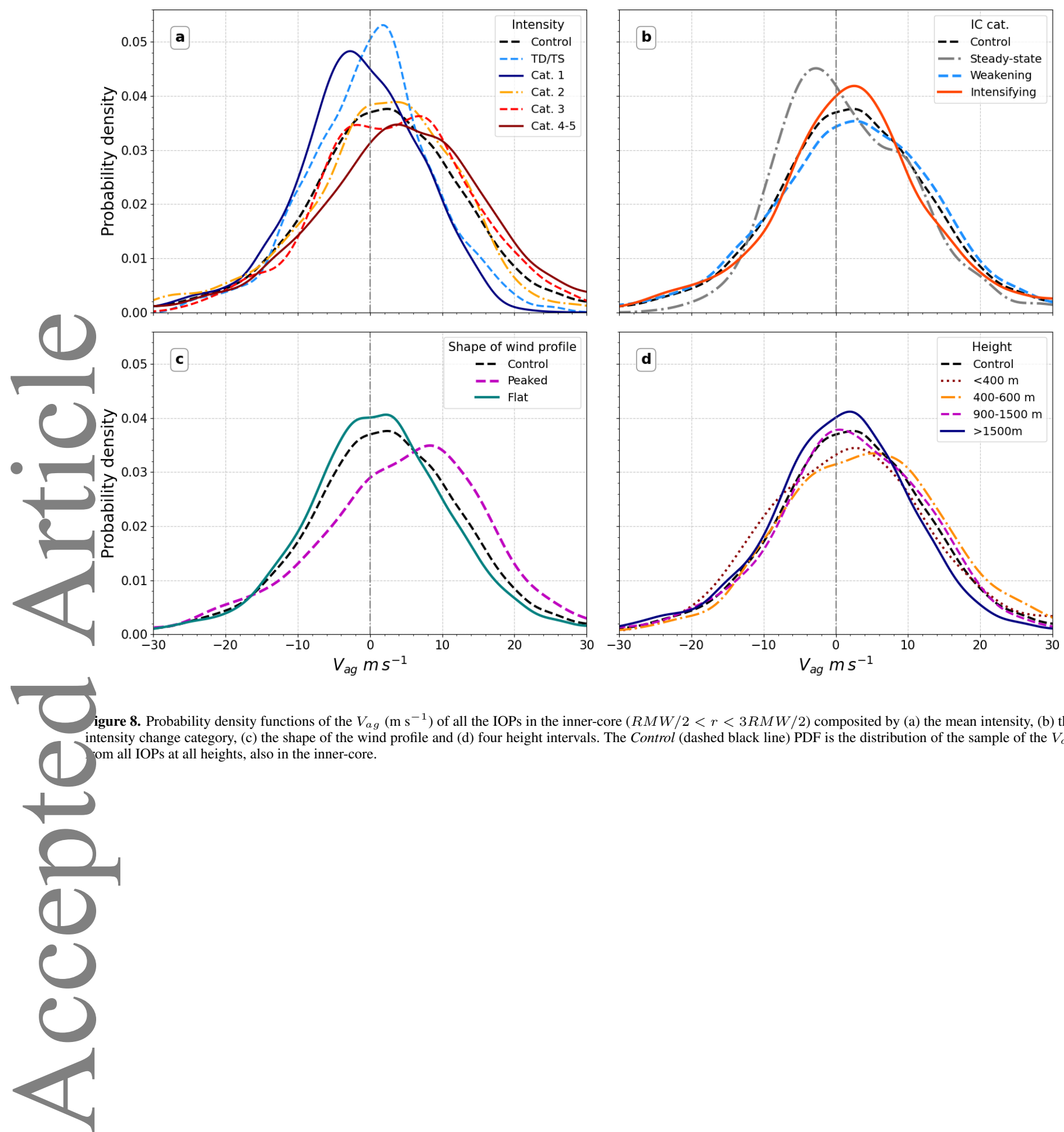

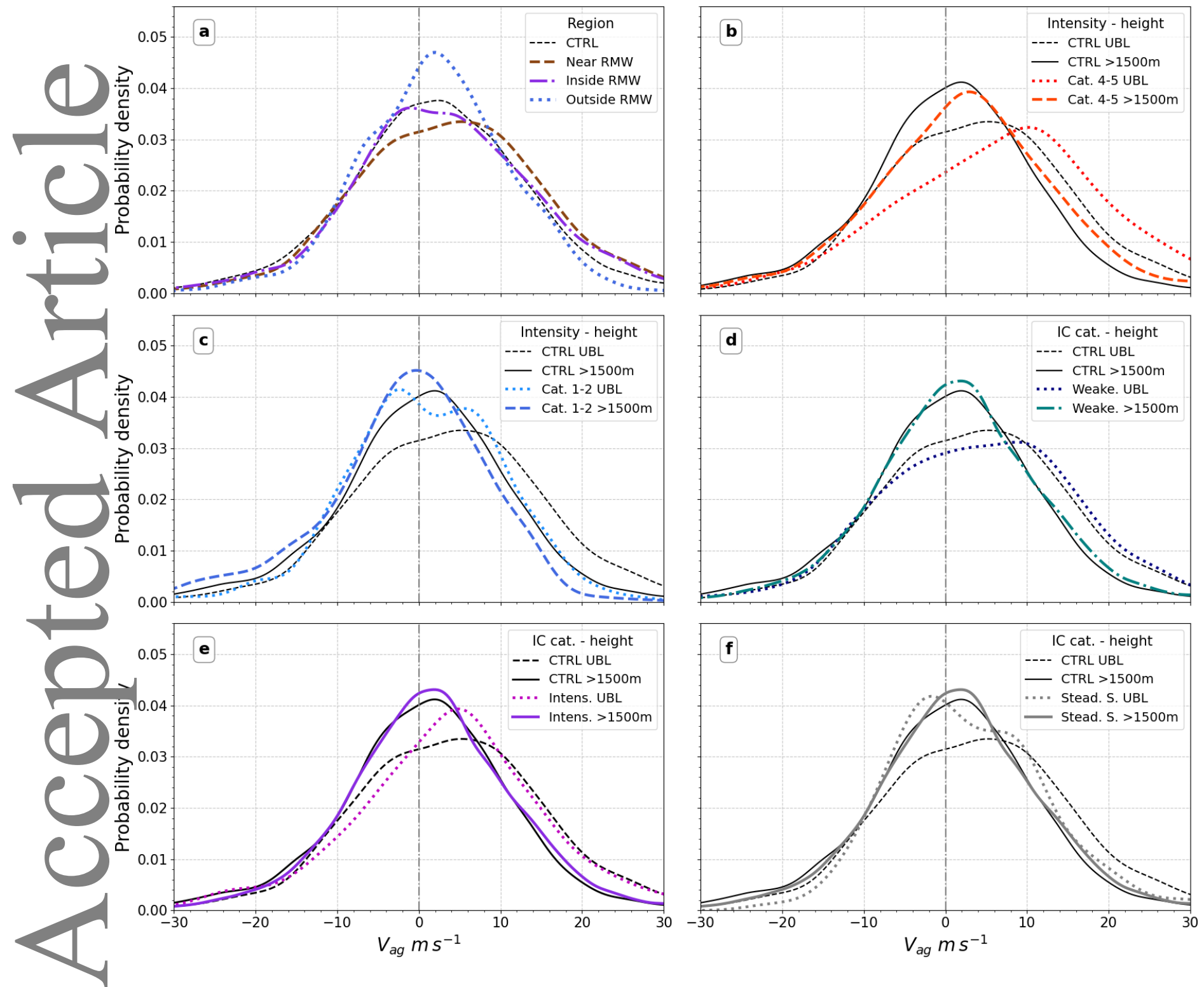

Figure 9. PDFs as in Figure 8. (a) shows the effect of the radial distance to storm centre by compositing for observations near the RMW ( $R M W / 2<r<3 R M W / 2$ ), inside the RMW $(0<r<R M W / 2)$, and outside the RMW $(r>3 R M W / 2)$. These PDFs, as well as the Control in this panel only contain observations in the interval of 400-600 m, the upper boundary layer (UBL). (b) PDFs of inner-core observations in Cat. 4-5 IOPs in the UBL (400-600 m) and the observations above $1500 \mathrm{~m}$. (c) is as in (b) but for Cat. 1-2 IOPs. (d, e, f) as in (b) but for Intensity change (IC) categories: weakening, intensifying and steady-state storms. The Control was also subsampled at these two height intervals in (b-f). 


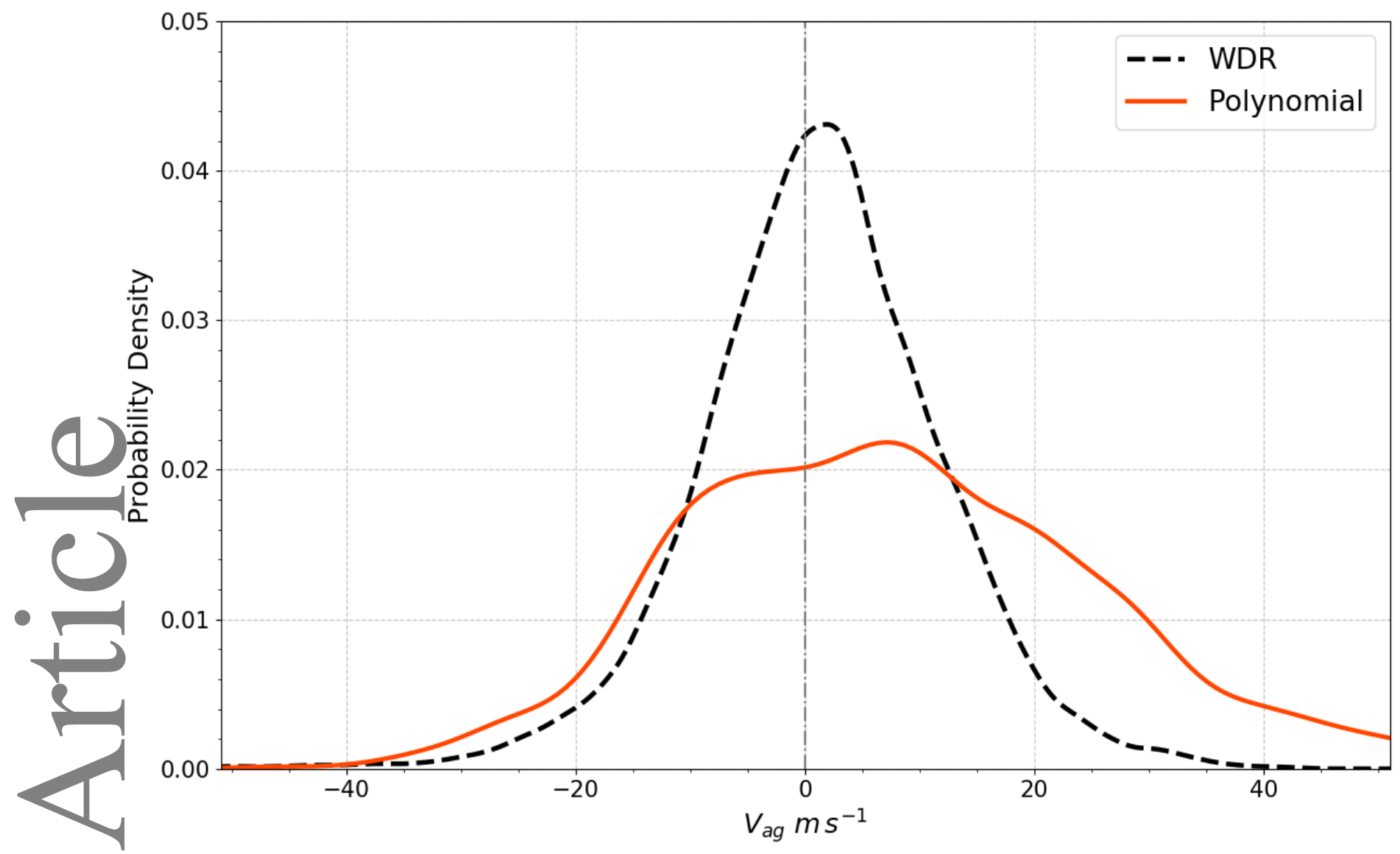

Figure 10. PDFs of gradient wind anomalies $V_{a g}$ computed from the WDR function (black dashed line) and the polynomial fit (orange solid line) at all height levels for Jil IOPs. The WDR function PDF is the same as the Control in the previous figures (e.g. Figure 8). 


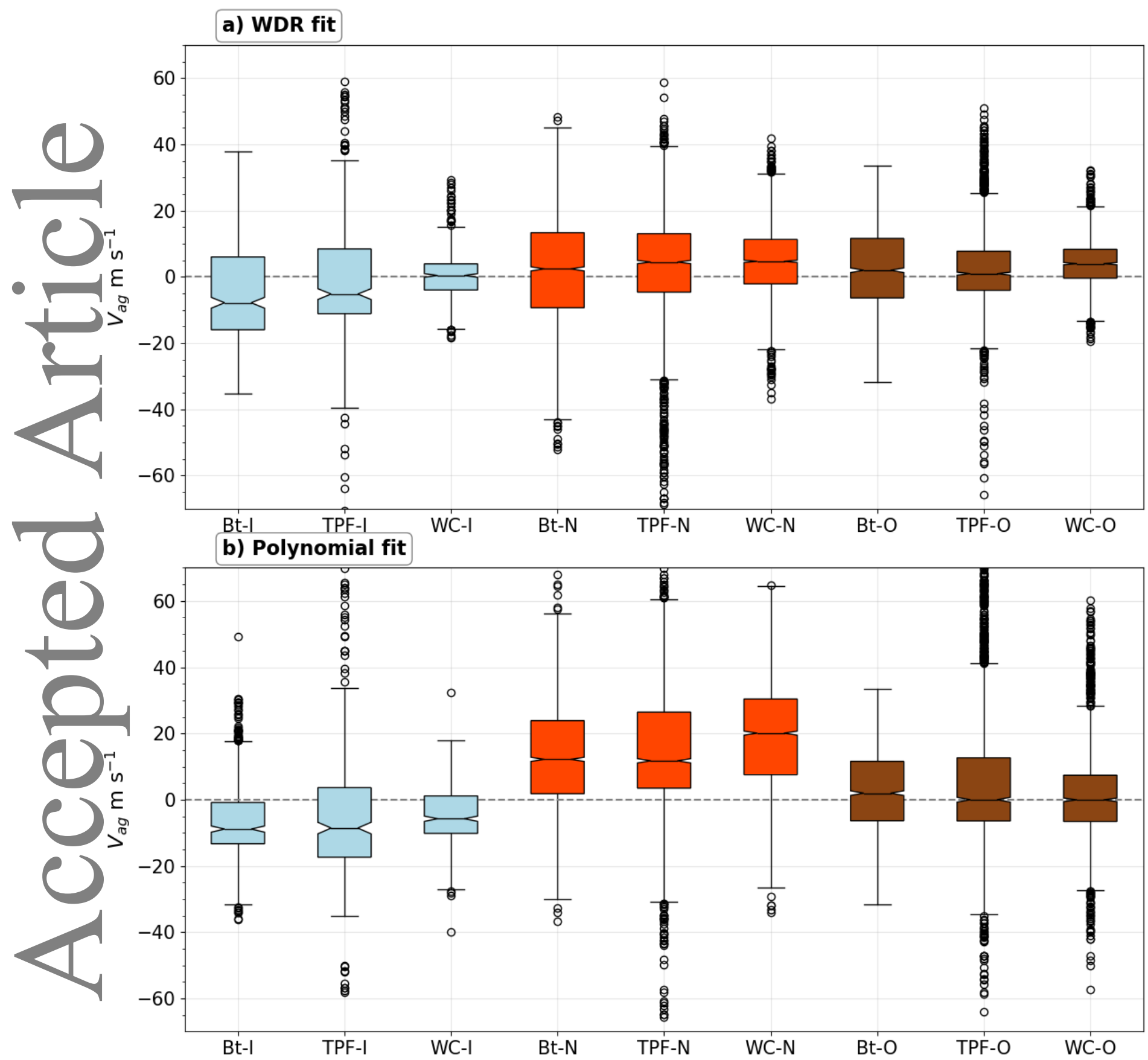

Figure 11. Boxplots of gradient wind balance anomalies ( $\left.V_{a g}\right)$ computed using the best-track (BT), the translating pressure fit (TPF) and the Willougby-Chelmow (WC) track finding algorithms in three different radial regions: inside (I, blue), near (N, red) and outside (O, maroon) the RMW. The boxplots are shown using (a) the WDR function and (b) the polynomial fit. 
Table 1. Information on the intense-observation periods (IOPs) used in this study. The table reports the start and end dates, the mean intensity $\bar{I}(k t)$, the intensity category (I. Cat.), the intensity change rate $\left(I C R ; k t(24 \mathrm{~h})^{-1}\right)$, the number of dropsondes in the inner-core (Drops), and whether the shape of the wind profile (WPS) was found to be peaked (P) or flat/non-peaked (NP) in (BL) or above (ABL) the boundary layer.

IOP Storm Start date hour End date hour $\quad \bar{I}(\mathrm{kt}) \quad$ I. Cat. ICR $(k t / 24-\mathrm{h}) \quad$ Drops $\quad$ WPS $\quad$ WPS

\begin{tabular}{|c|c|c|c|c|c|c|c|c|c|}
\hline & & & & & & & & BL & $\mathrm{ABL}$ \\
\hline 1 & Irene & $1999-10-1520$ & 1999-10-1601 & 65.0 & Cat. 1 & -10.0 & 25 & NP & NP \\
\hline 2 & Bret & 1999-08-21 17 & $1999-08-2121$ & 105.0 & Cat. 3 & 70.0 & 13 & $\mathrm{P}$ & NP \\
\hline 3 & Bret & 1999-08-21 21 & $1999-08-2203$ & 105.0 & Cat. 3 & 10.0 & 12 & $\mathrm{P}$ & NP \\
\hline 4 & Floyd & 1999-09-12 18 & 1999-09-1302 & 125.0 & Cat. 4 & 20.0 & 19 & $\mathrm{P}$ & NP \\
\hline 5 & Floyd & 1999-09-13 17 & 1999-09-1401 & 120.0 & Cat. 4 & -20.0 & 36 & NP & NP \\
\hline 6 & Floyd & 1999-09-15 20 & 1999-09-1609 & 90.0 & Cat. 2 & -40.0 & 62 & NP & NP \\
\hline 7 & Dennis & 1999-08-26 18 & $1999-08-2621$ & 65.0 & Cat. 1 & -10.0 & 15 & NP & NP \\
\hline 8 & Dennis & 1999-08-26 21 & 1999-08-27 02 & 65.0 & Cat. 1 & 0.0 & 3 & NP & NP \\
\hline 9 & Dennis & 1999-08-29 18 & 1999-08-30 01 & 90.0 & Cat. 2 & -10.0 & 25 & NP & NP \\
\hline 10 & Gabrielle & 2001-09-15 17 & 2001-09-16 00 & 47.5 & TS/TD & 10.0 & 35 & NP & NP \\
\hline 11 & Humberto & 2001-09-2319 & 2001-09-2402 & 85.0 & Cat. 2 & -30.0 & 69 & NP & NP \\
\hline 12 & Humberto & 2001-09-24 18 & $2001-09-2500$ & 65.0 & Cat. 1 & 0.0 & 77 & NP & NP \\
\hline 13 & Michelle & 2001-11-03 18 & 2001-11-04 05 & 115.0 & Cat. 4 & 10.0 & 31 & $\mathrm{P}$ & $\mathrm{P}$ \\
\hline 14 & Michelle & 2001-11-04 11 & 2001-11-04 17 & 120.0 & Cat. 4 & -30.0 & 14 & $\mathrm{P}$ & NP \\
\hline 15 & Lili & 2002-09-30 18 & 2002-10-01 01 & 70.0 & Cat. 1 & 40.0 & 34 & NP & NP \\
\hline 16 & Lili & 2002-10-02 02 & 2002-10-02 11 & 100.0 & Cat. 3 & 45.0 & 39 & $\mathrm{P}$ & $\mathrm{P}$ \\
\hline 17 & Lili & 2002-10-02 17 & 2002-10-03 01 & 123.3 & Cat. 4 & -90.0 & 46 & $\mathrm{P}$ & $\mathrm{P}$ \\
\hline 18 & Isidore & 2002-09-21 18 & $2002-09-2200$ & 110.0 & Cat. 3 & 0.0 & 73 & $\mathrm{P}$ & NP \\
\hline 19 & Isidore & 2002-09-22 18 & 2002-09-23 01 & 105.0 & Cat. 3 & -100.0 & 27 & NP & NP \\
\hline 20 & Isabel & 2003-09-12 16 & 2003-09-12 21 & 140.0 & Cat. 5 & -20.0 & 77 & $\mathrm{P}$ & NP \\
\hline 21 & Isabel & 2003-09-13 16 & 2003-09-14 01 & 137.5 & Cat. 5 & -10.0 & 43 & NP & NP \\
\hline 22 & Isabel & 2003-09-14 16 & 2003-09-1500 & 135.0 & Cat. 4 & -30.0 & 76 & NP & NP \\
\hline 23 & Isabel & 2003-09-18 14 & 2003-09-18 19 & 87.5 & Cat. 2 & -80.0 & 30 & NP & $\mathrm{P}$ \\
\hline 24 & Fabian & 2003-09-02 17 & 2003-09-02 23 & 120.0 & Cat. 4 & -20.0 & 65 & NP & NP \\
\hline 25 & Fabian & 2003-09-03 17 & 2003-09-03 22 & 110.0 & Cat. 3 & -10.0 & 59 & NP & NP \\
\hline 26 & Jeanne & 2004-09-24 17 & $2004-09-2500$ & 85.0 & Cat. 2 & 10.0 & 30 & NP & NP \\
\hline 27 & Jeanne & 2004-09-25 18 & $2004-09-2602$ & 105.0 & Cat. 3 & -60.0 & 53 & NP & NP \\
\hline 28 & Ivan & 2004-09-09 16 & 2004-09-10 00 & 130.0 & Cat. 4 & -10.0 & 26 & $\mathrm{P}$ & $\mathrm{P}$ \\
\hline 29 & Ivan & 2004-09-12 06 & 2004-09-1300 & 133.7 & Cat. 4 & 20.0 & 47 & $\mathrm{P}$ & $\mathrm{P}$ \\
\hline 30 & Ivan & 2004-09-13 18 & 2004-09-14 00 & 140.0 & Cat. 5 & -30.0 & 34 & $\mathrm{P}$ & $\mathrm{P}$ \\
\hline 31 & Ivan & 2004-09-14 06 & 2004-09-1500 & 121.6 & Cat. 4 & 0.0 & 50 & $\mathrm{P}$ & $\mathrm{P}$ \\
\hline 32 & Ivan & 2004-09-1500 & 2004-09-15 12 & 117.5 & Cat. 4 & -10.0 & 21 & NP & NP \\
\hline 33 & Ivan & 2004-09-15 17 & 2004-09-16 05 & 112.5 & Cat. 3 & -80.0 & 68 & NP & NP \\
\hline 34 & Frances & 2004-08-30 17 & $2004-08-3100$ & 110.0 & Cat. 3 & 20.0 & 21 & $\mathrm{P}$ & $\mathrm{P}$ \\
\hline 35 & Frances & 2004-08-31 16 & 2004-08-31 19 & 125.0 & Cat. 4 & -10.0 & 17 & NP & NP \\
\hline
\end{tabular}

This article is protected by copyright. All rights reserved. 
Frances

2004-09-01 16

2004-09-02 00

120.0

Cat. $4 \quad 10.0$

Frances

2004-09-02 17 2004-09-03 00

Cat. $3 \quad-30.0$

NP

NP

38

Frances

2004-09-03 15

2004-09-03 $23 \quad 90.0$

Cat. $2 \quad-5.0$

P

39

Ophelia

2005-09-09 $12 \quad$ 2005-09-09 18

TS/TD $\quad-10.0$

50

$\mathrm{P}$

40

Ophelia

2005-09-11 $11 \quad 2005-09-1201 \quad 65.0$

Cat. $1 \quad-10.0$

Rita

2005-09-21 20

2005-09-22 13

150.0

Cat. $5 \quad-60.0$

Rita

2005-09-22 13

2005-09-22 22

95.0

Cat. $2 \quad-20.0$

Rita

2005-09-22 22

2005-09-23 13

147.5

Cat. $5 \quad-10.0$

99

Rita

2005-09-23 13 2005-09-23 20

$128.3 \quad$ Cat. $4 \quad-20.0$

Rita

2005-09-23 20

2005-09-24 08

$113.3 \quad$ Cat. $3 \quad-80.0$

80

Ophelia

2005-09-14 $14 \quad 2005-09-1500 \quad 75.0$

Cat. $1 \quad-10.0$

Dennis

2005-07-08 $07 \quad 116.6$

Cat. $4 \quad 20.0$

Dennis

2005-07-09 06

2005-07-09 $13 \quad 80.0$

Cat. $1 \quad 60.0$

Katrina

2005-08-27 14

2005-08-28 01

$100.0 \quad$ Cat. $3 \quad 50.0$

Katrina

2005-08-28 17

2005-08-29 11

$131.2 \quad$ Cat. $4 \quad-65.0$

TS/TD $\quad-10.0$

Cat. $2 \quad 30.0$

Helene

2006-09-17 14

2006-08-31 19

60.0

Cat. 20.0

Helene

2006-09-19 16

2006-09-19 $20 \quad 90.0$

TS/TD $\quad 20.0$

Dolly

2008-07-22 08

2008-07-22 $14 \quad 55.0$

TS/TD $\quad 20.0$

Dolly

2008-07-22 23

2008-07-23 $02 \quad 65.0$

Cat. $1 \quad 40.0$

Gustav

2008-08-31 06

2008-09-01 0196.6

Cat. $2 \quad 0.0$

Gustav

2008-09-01 01

2008-09-01 $19 \quad 91.25$

Cat. $2 \quad-70.0$

\section{1}

17

46

60

NP

NP

NP NP

NP NP

NP NP

$\mathrm{P}$

$\mathrm{P}$

3

NP

99

NP

P

P

NP

NP

P NP

5

NP

NP

NP NP

17

NP NP

NP NP

60

$$
\mathrm{P}
$$$$
\mathrm{P}
$$

NP NP

NP P

NP

NP

NP

NP

NP

NP

62

2008-09-06 $14 \quad 2008-09-0700 \quad 115.0$

Cat. $4 \quad 0.0$

NP

NP

NP NP

64

2008-09-07 17

2008-09-08 $00 \quad 110.0$

Cat. $3 \quad-60.0$

$\mathrm{P}$

$\mathrm{P}$

$\mathrm{P}$

22 


\begin{tabular}{|c|c|c|c|c|c|c|c|c|c|}
\hline 76 & Earl & 2010-09-02 22 & 2010-09-03 13 & 90.0 & Cat. 2 & -30.0 & 34 & NP & NP \\
\hline 77 & Earl & 2010-09-03 13 & 2010-09-04 03 & 68.3 & Cat. 1 & -20.0 & 42 & NP & NP \\
\hline 78 & Bill & 2009-08-19 20 & 2009-08-20 02 & 115.0 & Cat. 4 & -20.0 & 22 & NP & NP \\
\hline 79 & Bill & 2009-08-20 08 & 2009-08-20 13 & 105.0 & Cat. 3 & 10.0 & 21 & NP & NP \\
\hline 80 & Hilary & 2011-09-28 17 & 2011-09-28 20 & 70.0 & Cat. 1 & -20.0 & 27 & $\mathrm{P}$ & NP \\
\hline 81 & Hilary & 2011-09-29 16 & 2011-09-29 19 & 50.0 & TS/TD & -40.0 & 16 & $\mathrm{P}$ & $\mathrm{P}$ \\
\hline 82 & Rina & $2011-10-2623$ & 2011-10-27 02 & 80.0 & Cat. 1 & -40.0 & 23 & NP & NP \\
\hline 83 & Isaac & $2012-08-2620$ & 2012-08-27 01 & 50.0 & TS/TD & 10.0 & 26 & NP & NP \\
\hline 84 & Isaac & 2012-08-28 11 & $2012-08-2815$ & 65.0 & Cat. 1 & 10.0 & 24 & NP & $\mathrm{P}$ \\
\hline 85 & Isaac & $2012-08-2820$ & 2012-08-2908 & 70.0 & Cat. 1 & -10.0 & 34 & NP & NP \\
\hline 86 & Isaac & 2012-08-27 04 & 2012-08-27 17 & 58.3 & TS/TD & 10.0 & 24 & $\mathrm{P}$ & $\mathrm{P}$ \\
\hline 87 & Isaac & 2012-08-27 16 & $2012-08-2803$ & 58.3 & TS/TD & 10.0 & 34 & NP & NP \\
\hline 88 & Sandy & $2012-10-2521$ & $2012-10-2600$ & 75.0 & Cat. 1 & -20.0 & 11 & NP & NP \\
\hline 89 & Sandy & $2012-10-2600$ & $2012-10-2604$ & 65.0 & Cat. 1 & -20.0 & 14 & NP & NP \\
\hline 90 & Sandy & 2012-10-26 09 & $2012-10-2614$ & 65.0 & Cat. 1 & -10.0 & 21 & NP & NP \\
\hline 91 & Sandy & $2012-10-2708$ & 2012-10-27 14 & 70.0 & Cat. 1 & -10.0 & 27 & NP & NP \\
\hline
\end{tabular}


Table 2. Parameter limits for the pressure fit using the piece-wise function of Willoughby et al. (2006). For the profile inside the radius of maximum wind, $n$ is the degree of the polynomial and $V_{\max }$ and $R_{\max }$ are the maximum wind speed and radius of maximum wind, respectively. $L_{1}$ and $L_{2}$ are length scales that constrain the exponential function outside the radius of maximum wind, and $R_{1}$ and $R_{2}$ are the radial distances that determine where the inner, transition and outer profiles are defined.

\begin{tabular}{cccc} 
Parameter & Lower limit & Upper limit & Unit \\
\hline \hline$n$ & 1 & 1.5 & N/A \\
$L_{1}$ & 420 & 700 & $\mathrm{~km}$ \\
$L_{2}$ & 15 & 35 & $\mathrm{~km}$ \\
$R_{1}$ & $\mathrm{RMW}-(\mathrm{RMW} / 2)$ & $\mathrm{RMW}$ & $\mathrm{km}$ \\
$R_{2}$ & $\mathrm{RMW}$ & $2 \mathrm{RMW}$ & $\mathrm{km}$ \\
$R_{\max }$ & $\mathrm{RMW}-2$ & $\mathrm{RMW}+2$ & $\mathrm{~km}$ \\
$V_{\max }$ & $V_{\max }-10$ & $V_{\max }+10$ & $m s^{-1}$ \\
\hline
\end{tabular}

Table 3. Statistical information of each composite of inner-core observations reporting the: mean and standard deviation and the p-value of a Welch and Kolmogorov-Smirnov (KS) statistical tests. Each test compared each composite to the Control sample, which includes all inner-core observations. The probabilities $p$ of the agradient wind being higher than $15 \mathrm{~m} \mathrm{~s}^{-1}$ and lower than $-15 \mathrm{~m} \mathrm{~s}^{-1}$ in each distribution are also shown.

\begin{tabular}{ccccccc}
\hline Composite & Mean & st dev & p-value (Welch) & p-value $(\mathrm{KS})$ & $p\left(V_{a g}<-15 \mathrm{~ms}^{-1}\right)$ & $p\left(V_{a g}>15 \mathrm{~ms}^{-1}\right)$ \\
\hline Control & 1.3 & 12.1 & - & - & 0.07 & 0.11 \\
\hline Intensifying & 1.08 & 11.8 & $>0.05$ & $<0.05$ & 0.08 & 0.11 \\
Weakening & 1.5 & 12.6 & $>0.05$ & $>0.05$ & 0.08 & 0.14 \\
Steady-state & 0.8 & 8.7 & $>0.05$ & $<0.05$ & 0.03 & 0.07 \\
\hline TS/TD & -1.0 & 8.0 & $>0.05$ & $<0.01$ & 0.05 & 0.05 \\
Cat. 1 & -2.4 & 8.5 & $<0.01$ & $<0.01$ & 0.07 & 0.03 \\
Cat. 2 & 0.1 & 10.7 & $<0.05$ & $<0.01$ & 0.1 & 0.06 \\
Cat. 3 & 3.0 & 11.0 & $<0.01$ & $<0.01$ & 0.06 & 0.14 \\
Cat. 4,5 & 3.9 & 12.8 & $<0.01$ & $<0.01$ & 0.07 & 0.19 \\
\hline Flat & 0.3 & 11.2 & $<0.01$ & $<0.01$ & 0.09 & 0.09 \\
Peaked & 3.4 & 13.5 & $<0.01$ & $<0.01$ & 0.07 & 0.19 \\
\hline 400-600 m & 2.6 & 12.7 & $<0.01$ & $<0.01$ & 0.08 & 0.16 \\
$>1500 \mathrm{~m}$ & -0.7 & 11.0 & $<0.01$ & $<0.01$ & 0.1 & 0.08 \\
\hline
\end{tabular}

